\title{
PARAMETER ROBUST PRECONDITIONING BY CONGRUENCE FOR MULTIPLE-NETWORK POROELASTICITY*
}

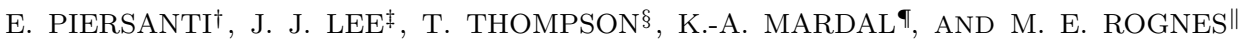

\begin{abstract}
The mechanical behavior of a poroelastic medium permeated by multiple interacting fluid networks can be described by a system of time-dependent partial differential equations known as the multiple-network poroelasticity (MPET) equations or multiporosity/multipermeability systems. These equations generalize Biot's equations, which describe the mechanics of the one network case. The efficient numerical solution of the MPET equations is challenging, in part due to the complexity of the system and in part due to the presence of interacting parameter regimes. In this paper, we present a new strategy for efficiently and robustly solving the MPET equations numerically. In particular, we discuss an approach to formulating finite element methods and associated preconditioners for the MPET equations based on simultaneous diagonalization of the element matrices. We demonstrate the technique for the multicompartment Darcy equations, with large exchange variability, and the MPET equations for a nearly incompressible medium with large exchange variability. The approach is based on designing transformations of variables that simultaneously diagonalize (by congruence) the equations' key operators and subsequently constructing parameter-robust block diagonal preconditioners for the transformed system. The proposed approach is supported by theoretical considerations as well as by numerical results.
\end{abstract}

Key words. preconditioning, poroelasticity, parameter-robust

AMS subject classifications. 65M55, 65M60

DOI. $10.1137 / 20 \mathrm{M} 1326751$

1. Introduction. In this paper, we consider the preconditioned iterative solution of finite element discretizations of the multiple-network poroelasticity (MPET) equations. These equations traditionally originate in geomechanics where they are also known under the term multiporosity/multipermeability systems [1]. The MPET equations generalize Biot's equations [2] from the one network to the multiple network case, and multicompartment Darcy (MPT) equations [19] from a porous (but rigid) to a poroelastic medium. Over the last decade, the MPT and MPET equations have seen a surge of interest in biology and physiology, e.g., to model perfusion in the heart [19, 14], brain [13], liver [3], or in cancer [25], or to model the interaction between elastic deformation and fluid flow and transport in the brain [5, 6, 21, 28, 29, 30].

Concretely, the quasi-static MPET equations read as follows [1]: for a given number of networks $J \in \mathbb{N}$, find the displacement $u$ and the network pressures $p_{j}$ for

*Submitted to the journal's Computational Methods in Science and Engineering section March 23, 2020; accepted for publication (in revised form) April 14, 2021; published electronically August 4, 2021.

https://doi.org/10.1137/20M1326751

Funding: The work of the second author was supported by the European Research Council under the European Union's Seventh Framework Programme (FP7/2007-2013) ERC grant agreement 339643. The work of the third, fourth, and fifth authors was supported by the Research Council of Norway under the FRINATEK Young Research Talents Programme through project 250731/F20 (Waterscape).

†Simula Research Laboratory, P. O. Box 134, 1325 Lysaker, Norway (eleonora@simula.no).

${ }^{\ddagger}$ Department of Mathematics, Baylor University, One Bear Place \# 97328, Waco, TX 78798 USA (jeonghun_lee@baylor.edu).

§Simula Research Laboratory, Norway. Current address: Mathematical Institute, Oxford University, Oxford, United Kingdom (thompsont@maths.ox.ac.uk).

"Department of Mathematics, University of Oslo, P. O. Box 1053 Blindern, 0316 Oslo, Norway and Simula Research Laboratory, P. O. Box 134, 1325 Lysaker, Norway (kent-and@simula.no).

" Simula Research Laboratory, P. O. Box 134, 1325 Lysaker, Norway (meg@simula.no).

B984 
TABLE 1

Sample parameter values for hydraulic conductivities and exchange coefficients with reference to (1.1), and/or (1.2).

\begin{tabular}{lrrr}
\hline Parameter & Unit & Value & Reference \\
\hline Hydraulic conductivities $\left(K_{j}\right)$ & $\mathrm{mm}^{2}(\mathrm{kPa} \mathrm{s})^{-1}$ & & \\
\hline Brain gray matter & & $2.0 \times 10^{-3}$ & {$[27]$} \\
Brain white matter & & $2.0 \times 10^{-2}$ & {$[27]$} \\
Cardiac arteries & & 1.0 & {$[19]$} \\
Cardiac capillaries & 2.0 & {$[19]$} \\
Cardiac veins & 10.0 & {$[19]$} \\
Brain vasculature & & $3.75 \times 10^{1}$ & {$[30]$} \\
Brain fluid exchange & & $1.57 \times 10^{-2}$ & {$[30]$} \\
\hline Exchange coefficients $\left(\xi_{j \rightarrow i}\right)$ & $(\mathrm{kPa} \mathrm{s})^{-1}$ & & \\
\hline Brain capillary-vasculature & & $1.5 \times 10^{-16}$ & {$[30]$} \\
Brain capillary-tissue fluid & & $2.0 \times 10^{-16}$ & {$[30]$} \\
Brain tissue fluid-veins & & $2.0 \times 10^{-10}$ & {$[30]$} \\
Cardiac capillary-arteries & & $2.0 \times 10^{-2}$ & {$[19]$} \\
Cardiac capillary-veins & & $5.0 \times 10^{-2}$ & {$[19]$} \\
\hline
\end{tabular}

$j=1, \ldots, J$ such that

$$
\begin{aligned}
-\operatorname{div}(2 \mu \varepsilon(u)+\lambda \operatorname{div} u \mathbb{I})+\sum_{j} \alpha_{j} \nabla p_{j} & =f, \\
s_{j} \dot{p}_{j}+\alpha_{j} \operatorname{div} \dot{u}-\operatorname{div} K_{j} \nabla p_{j}+\sum_{i} \xi_{j \leftarrow i}\left(p_{j}-p_{i}\right) & =g_{j},
\end{aligned}
$$

where $u=u(x, t), p_{j}=p_{j}(x, t)$ for $x \in \Omega \subset \mathbb{R}^{d}(d=1,2,3), t \in(0, T]$, and $\mathbb{I}$ is the $d \times d$ identity matrix. Physically, (1.1) describe a porous and elastic medium permeated by a number of fluid networks under the assumptions that the solid matrix can be modeled as isotropic and linearly elastic with Lamé constants $\mu>0$ and $\lambda>0$, and the transfer between the networks is regulated by the corresponding pressure differences with exchange coefficients $\xi_{j \leftarrow i} \geq 0$. For each network $j$, we define the Biot-Willis coefficient $\alpha_{j} \in(0,1]$ such that $\sum_{j} \alpha_{j} \leq 1$, the storage coefficient $s_{j}>0$, and the hydraulic conductivity tensor $K_{j}=\kappa_{j} / \nu_{j}>0$ with $\kappa_{j}$ and $\nu_{j}$ being the permeability and fluid viscosity, respectively. Moreover, $\nabla$ denotes the column-wise gradient, $\varepsilon$ is the symmetric gradient, div denotes the (rowwise) divergence, the superposed dot denotes the time derivative(s), and $I$ denotes the identity matrix. On the right-hand side, $f$ represents body forces and $g_{j}$ sources (or sinks) in network $j$ for $j=1, \ldots, J$.

The MPT equations represent a reduced version of (1.1) that results from ignoring the elastic contribution of the solid matrix. These equations then read as follows: for a given number of networks $J \in \mathbb{N}$, find the network pressures $p_{j}$ for $j=1, \ldots, J$ such that

$$
-\operatorname{div} K_{j} \nabla p_{j}+\sum_{i=1}^{J} \xi_{j \leftarrow i}\left(p_{j}-p_{i}\right)=g_{j}
$$

where for $i, j=1, \ldots, J, p_{j}=p_{j}(x)$ for $x \in \Omega \subset \mathbb{R}^{d}(d=1,2,3)$, the parameters $K_{j}$ and $\xi_{j \leftarrow i}$ remain the hydraulic conductivity and exchange coefficients, respectively, and $g_{j}$ again represents other sources (or sinks) in each network. The relative size of the conductivities $K_{j}$ and the exchange coefficients $\xi_{j \leftarrow i}$ may vary tremendously in applications. Large parameter variation is certainly present in applied problems of a physiological nature; a selection of representative parameter values, from research literature, is given in Table 1. Here, we see that the hydraulic conductivities span four orders of magnitude while the exchange coefficients span fourteen orders of magnitude. 
Hence, there is a need for preconditioners that are robust with respect to variations in parameters. Physiological applications, in particular, can benefit from preconditioners which are robust with respect to $K_{j}, \xi_{j \leftarrow i}$, and $\lambda$ as in (1.1) and (1.2).

With this in mind, parameter-robust numerical approximations and solution algorithms for (1.1) are currently active research topics. In the nearly incompressible case $\lambda \gg 1$, the standard two-field variational formulation of (1.1) is not robust. To address this challenge, we introduced and analyzed a mixed finite element method for the MPET equations based on a total pressure formulation in [17]. We note that the total pressure in the case of one network was presented in [16, 22]. Hong et al. [9] shortly thereafter extended the three-field formulation in [8] to parameterrobust MPET equations taking the displacement, the network fluid fluxes, and the network pressures as unknowns. In fact, the approach of [9] is the first result advancing preconditioners which are robust with respect to every material and discretization parameter, including the network transfer terms we consider here. As an alternative to these fully coupled approaches a form of splitting scheme has been analyzed by Lee [15]. Regarding the iterative solution and preconditioning of the fully coupled formulations, a robust preconditioner for Biot's equations (the case for $J=1$ ) was presented by Lee, Mardal, and Winther [16]. Hong et al. [9] presented both theoretical results and numerical examples regarding parameter-robust preconditioners for the MPET equations with their extended three-field-type formulation. Hong et al. further developed parameter-robust solver algorithms, an iterative solver algorithm using the iterative coupling approach (cf. [20]) in [11], and an Uzawa-type algorithm in [10].

In this paper, we present a parameter-robust preconditioning approach for linear systems of equations resulting from a conforming finite element discretization of the total pressure variational formulation of the MPET equations. The main focus of this work is to achieve preconditioners which are robust for large ratios of exchange coefficients. The potential of diagonalization by congruence was also mentioned by Hong et al. [9, Remark 6]. In this manuscript, we discuss diagonalization by congruence as a general method in the context of preconditioning the MPET equations. The key idea is, as introduced for the MPT equations in [23], to design a parameter-dependent transformation of the pressure variables $p=\left(p_{1}, \ldots, p_{J}\right)$ into a set of transformed variables $\tilde{p}$. The transformation should be such that the originally coupled exchange operator decouples while the originally decoupled diffusion operator remains decoupled (i.e., remains diagonal). We will discuss this approach, and its associated matrix theory in detail. We illustrate the use of the method to construct parameter-robust preconditioners for both the MPT equations (section 3) and the MPET equations (section 4).

There are several notable differences between the approach of the current work and that of [9]. First, the formulation of [9] introduces $2 J+1$ unknowns for a multiple poroelasticity system consisting of $J$ fluid networks. Conversely, the total pressure formulation, which we use here, requires $J+2$ unknowns. Thus, our approach can confer a significant computational savings for applications where $J$ is appreciably large. Second, the spatial discretization in [9] employs several $H$ (div) finite element spaces; as a result, multiple $H$ (div) preconditioners are needed for robust preconditioning. Conversely, we discretize by means of continuous Galerkin finite elements; as a result, block preconditioners can be constructed using typical preconditioners for second order elliptic operators. It should be noted, though, that our approach does not satisfy local mass conservation; the paradigm of [9] is locally mass conservative, due to the $H$ (div) elements, with the price being an increase in computational cost. Third, the 
preconditioning results of [9] are unequivocally robust for all parameter ranges; a first result of its kind for generalized poroelasticity. Conversely, our approach requires a slight restriction, in general, of the parameter ranges for the network storage coefficients; this restriction can be removed, however, for materials with nondegenerate storage coefficients satisfying $\lambda^{-1} \lesssim s_{j}$ (cf. Remark 4.4). Overall, if the number of networks is small, local mass conservation is important for the application or, if the material storage coefficients are degenerate then [9] is a strong option. Conversely, if local mass conservation is not a strict application concern and the network storage coefficients are not degenerate, our approach confers a significant computational advantage, over that of [9] when the number of fluid networks $(J)$ is large.

This manuscript is organized as follows. We introduce notation and review relevant preconditioning and matrix theory in section 2 . We briefly consider the reduced case of the MPT equations in section 3 before turning to the analysis of the preconditioner for the MPET equations in section 4. Finally, we present some conclusions and outlook in section 5 .

2. Preliminaries. In section 2.2 we briefly review preconditioning of parameterdependent systems and state a known result regarding simultaneous diagonalization by congruence. Notation for the remainder of the manuscript is discussed in section 2.1 .

2.1. Notation. In the subsequent manuscript, we use the following notation. Let $\Omega$ be an open, bounded domain in $\mathbb{R}^{d}, d=2,3$, with Lipschitz polyhedral boundary $\partial \Omega$. We denote by $L^{2}(\Omega)$ the space of square integrable functions on $\Omega$ with inner product $\langle\cdot, \cdot\rangle$ and norm $\|\cdot\|$. We denote by $H^{m}(\Omega)$ the standard Sobolev space with norm $\|\cdot\|_{H^{m}}$ and seminorm $|\cdot|_{H^{m}}$ for $m \geq 1$ and $H^{m}\left(\Omega ; \mathbb{R}^{d}\right)$ the corresponding $d$-vector fields. We use $H_{0}^{m}(\Omega)$ to denote the subspace of $H^{m}(\Omega)$ with vanishing trace on the boundary of $\Omega$. Let $\Gamma$ be a subset of $\partial \Omega$ such that $\partial \Omega \backslash \Gamma$ has a positive $(d-1)$-dimensional Lebesgue measure. $H_{\Gamma}^{m}(\Omega)$ is the subspace of $H^{m}(\Omega)$ such that the elements in $H_{\Gamma}^{m}(\Omega)$ have vanishing trace on $\Gamma . H_{\Gamma}^{m}\left(\Omega ; \mathbb{R}^{d}\right)$ is the subspace of $H^{m}\left(\Omega ; \mathbb{R}^{d}\right)$ such that every $v_{j}$ in $\left(v_{1}, \ldots, v_{d}\right) \in H^{m}\left(\Omega ; \mathbb{R}^{d}\right)$ is an element in $H_{\Gamma}^{m}(\Omega)$. Throughout this paper we set $\Gamma$ as a fixed subset of $\partial \Omega$ satisfying the aforementioned assumption.

We introduce the parameter-dependent $L^{2}$-inner product and norm:

$$
\|p\|_{\beta}^{2}=\langle p, p\rangle_{\beta}=\langle\beta p, p\rangle
$$

for $\beta \in L^{\infty}(\Omega), \beta(x)>0$, and $p \in L^{2}(\Omega)$ (and similarly for vector or tensor fields). The notation II will denote an identity $d \times d$ matrix while $I_{V}$ will denote the identity operator on a Hilbert space $V$. To be self-contained we recall the Kronecker product of matrices. If $A$ in $\mathbb{R}^{m \times n}$ and $B \in \mathbb{R}^{r \times s}$ are two real-valued matrices then $A \otimes B$ is the $m r \times n s$ matrix defined by multiplying each entry of $A$ by the matrix $B$. That is,

$$
A \otimes B=\left[\begin{array}{cccc}
a_{11} B & a_{12} B & \ldots & a_{1 n} B \\
a_{21} B & a_{22} B & \ldots & a_{2 n} B \\
\vdots & \ddots & \ddots & \vdots \\
a_{m 1} B & a_{m 2} B & \ldots & a_{m n} B
\end{array}\right]
$$

We can consider its natural extension for a matrix $A$ and a linear operator $B$. More specifically, if $W$ is a Hilbert space, $Q$ is the $n$-fold product $Q=W \times W \times \cdots \times W, A$ is an $n \times n$ matrix, and $B$ is a linear operator on $W$, then $A \otimes B$ is the linear operator on $Q$ defined by (2.1). 
Finally, we introduce a notation for uniform proportionality, used throughout the manuscript, as

$$
X \lesssim Y
$$

That is, $X \lesssim Y$ implies the existence of some real constant $c_{0}>0$ such that $X \leq c_{0} Y$; any relationship between $c_{0}$ and pertinent mathematical objects, such as the total number of porous media networks considered, will be specified.

2.2. Preconditioning of parameter-dependent systems. In this paper, we consider the preconditioning of discretizations of the systems (1.1) and (1.2) under large parameter variations. Therefore, we begin by summarizing core aspects of the theory of parameter-robust preconditioning as presented in [18]. We will apply this theory for formulations of the MPT equations (1.2) and MPET equations (1.1) in the subsequent sections.

Let $X$ be a separable, real Hilbert space with inner product $\langle\cdot, \cdot\rangle_{X}$, norm $\|\cdot\|_{X}$, and dual space $X^{*}$. Let $\mathcal{A}: X \rightarrow X$ be an invertible, symmetric isomorphism on $X$ such that $\mathcal{A} \in \mathcal{L}\left(X, X^{*}\right)$, where $\mathcal{L}\left(X, X^{*}\right)$ is the set of bounded linear operators mapping $X$ to its dual. Given $f \in X^{*}$ consider the problem of finding $x \in X$ such that

$$
\mathcal{A} x=f .
$$

The preconditioned problem reads as follows,

$$
\mathcal{B A} x=\mathcal{B} f,
$$

where $\mathcal{B} \in \mathcal{L}\left(X^{*}, X\right)$ is a symmetric isomorphism defining the preconditioner. The convergence rate of a Krylov space method for this problem can be bounded in terms of the condition number $\kappa(\mathcal{B A})$, where

$$
\kappa(\mathcal{B A})=\|\mathcal{B} \mathcal{A}\|_{\mathcal{L}(X, X)}\left\|(\mathcal{B A})^{-1}\right\|_{\mathcal{L}(X, X)} .
$$

Here, the operator norm $\|\mathcal{A}\|_{\mathcal{L}\left(X, X^{*}\right)}$ is defined by

$$
\|\mathcal{A}\|_{\mathcal{L}\left(X, X^{*}\right)}=\sup _{x \in X} \frac{\|\mathcal{A} x\|_{X^{*}}}{\|x\|_{X}} .
$$

Now, for a parameter $\varepsilon$ (or more generally a set of parameters $\varepsilon$ ) consider the parameter-dependent operator $\mathcal{A}_{\varepsilon}$ and its preconditioner $\mathcal{B}_{\varepsilon}$. Assume that we can choose appropriate spaces $X_{\varepsilon}$ and $X_{\varepsilon}^{*}$ such that the norms

$$
\left\|\mathcal{A}_{\varepsilon}\right\|_{\mathcal{L}\left(X_{\varepsilon}, X_{\varepsilon}^{*}\right)} \text { and }\left\|\mathcal{A}_{\varepsilon}^{-1}\right\|_{\mathcal{L}\left(X_{\varepsilon}^{*}, X_{\varepsilon}\right)}
$$

are bounded independently of $\varepsilon$. Similarly, we assume that we can find a preconditioner $\mathcal{B}_{\varepsilon}$ such that the norms $\left\|\mathcal{B}_{\varepsilon}\right\|_{\mathcal{L}\left(X_{\varepsilon}, X_{\varepsilon}^{*}\right)}$ and $\left\|\mathcal{B}_{\varepsilon}^{-1}\right\|_{\mathcal{L}\left(X_{\varepsilon}^{*}, X_{\varepsilon}\right)}$ are bounded independently of $\varepsilon$. Given these assumptions, the condition number $\kappa\left(\mathcal{B}_{\varepsilon} \mathcal{A}_{\varepsilon}\right)$ will be bounded independently of $\varepsilon$. We will refer to such a preconditioner as robust in (or with respect to) $\varepsilon$. We conclude this section with a change of variables result, recalled from basic matrix analysis [12], that will prove effective in the sections that follow.

Lemma 2.1 (diagonalization by congruence). Let $W$ be a real Hilbert space and $Q=W \times W \times \cdots \times W$ be the $n$-fold direct product of $W$ for a fixed $n \in \mathbb{N}$. Let $A: W \rightarrow W^{*}$ and $B: W \rightarrow W^{*}$ be linear operators. Suppose that $K, E \in \mathbb{R}^{n \times n}$ are 
symmetric matrices and that at least one of $K$ or $E$ is positive definite. Define the operators $S: Q \rightarrow Q^{*}$ and $T: Q \rightarrow Q^{*}$ by

$$
S=K \otimes A \quad \text { and } \quad T=E \otimes B,
$$

where $\otimes$ is the Kronecker product. Consider the variational problem given $f \in Q^{*}$ find $p=\left(p_{1}, p_{2}, \ldots, p_{n}\right)^{T} \in Q$ such that

$$
\langle S p, q\rangle+\langle T p, q\rangle=\langle f, q\rangle \quad \forall q \in Q,
$$

where $\langle\cdot, \cdot\rangle$ is the duality pairing of $Q^{*}$ and $Q$. Then there exists an invertible matrix $P \in \mathbb{R}^{n \times n}$ such that the above variational problem is equivalent to find $\tilde{p} \in Q$ such that

$$
\left\langle D_{S} \tilde{p}, q\right\rangle+\left\langle D_{T} \tilde{p}, q\right\rangle=\langle F, q\rangle \quad \forall q \in Q
$$

where $F=\left(P^{T} \otimes I_{W^{*}}\right) f$ for $I_{W^{*}}$ the identity operator on $W^{*}$, and $D_{S}=\left(P^{T} K P\right) \otimes A$ and $D_{T}=\left(P^{T} E P\right) \otimes B$ are block diagonal linear operators from $Q$ to $Q^{*}$.

Proof. Apply [12, Theorem 4.5.17a-b p. 287] the hypotheses on the matrices $K$ and $E$ and properties of the tensor product; see Appendix A for details.

3. Preconditioning the MPT equations via diagonalization. In this section, we present the method of simultaneous diagonalization by congruence, and demonstrate how the method can be applied to variational formulations and their associated preconditioners. Motivated by (1.1), we first consider the simpler MPT equations as in [23]. The core idea is to reformulate the MPT equations using a change of pressure variables $p$. In particular, we aim to find a transformation of the variables $p \mapsto \tilde{p}$ such that the transformed system of pressure equations decouple. Here, we will consider a Hilbert space $W$ and the $J$-fold product $Q=W \times W \times \cdots \times W$. Each pressure $p_{j}$, for $j=1,2, \ldots, J$ satisfies $p_{j} \in W$ and we will write $p=\left(p_{1}, p_{2}, \ldots, p_{J}\right) \in Q$. In the sections that follow, we briefly illustrate the core idea, formulation of the MPT equations, and resulting preconditioner, and refer to [23] for more details. This approach is then extended to the MPET equations in section 4.

3.1. The MPT equations in operator form. We consider the MPT equations as defined by (1.2). We further impose homogeneous Dirichlet boundary conditions for all pressures: $p_{j}=0$ on $\partial \Omega$ for $1 \leq j \leq J$. Define $\xi_{j}=\sum_{i=1}^{J} \xi_{j \leftarrow i}$ for each $1 \leq j \leq J$. Let us define two $J \times J$ matrices:

$$
K=\left(\begin{array}{cccc}
K_{1} & 0 & \cdots & 0 \\
0 & K_{2} & \cdots & 0 \\
\vdots & \vdots & \ddots & \vdots \\
0 & 0 & \cdots & K_{J}
\end{array}\right), \quad E=\left(\begin{array}{cccc}
\xi_{1} & -\xi_{1 \leftarrow 2} & \cdots & -\xi_{1 \leftarrow J} \\
-\xi_{1 \leftarrow 2} & \xi_{2} & \cdots & -\xi_{2 \leftarrow J} \\
\vdots & \vdots & \ddots & \vdots \\
-\xi_{1 \leftarrow J} & -\xi_{2 \leftarrow J} & \cdots & \xi_{J}
\end{array}\right)
$$

The system (1.2) can be expressed in operator form as given $g \in Q$ find $p \in Q$ satisfying

$$
\mathcal{A}_{\mathrm{MPT}} p=g, \quad \text { where } \quad \mathcal{A}_{\mathrm{MPT}}=-K \otimes \Delta+E \otimes I_{W} .
$$

In the above, $-K \otimes \Delta: Q \rightarrow Q^{*}$ is the block diagonal operator such that its $j$ th block is given by the bilinear form $\left\langle K_{j} \nabla p_{j}, \nabla q_{j}\right\rangle$ for $p_{j}, q_{j} \in Q_{j}=W$, and $E \otimes I_{W}: Q \rightarrow Q^{*}$ is the block operator such that its $(i, j)$-block $E_{i j}$ is defined by the bilinear forms

$$
-\left\langle\xi_{i \leftarrow j} p_{i}, q_{j}\right\rangle \text { if } i \neq j, \quad\left\langle\xi_{j} p_{j}, p_{j}\right\rangle \text { if } i=j .
$$


We note that $K$ is real, positive definite and diagonal (and thus invertible), and that $E$ is real, symmetric, and (weakly row) diagonally dominant by definition. In particular, $E$ is symmetric positive semidefinite because of the identity

$$
w E w^{T}=\sum_{1 \leq i, j \leq J} \xi_{i \leftarrow j}\left(w_{i}-w_{j}\right)^{2}
$$

for $w=\left(w_{1}, w_{2}, \ldots, w_{J}\right)$ with the convention $\xi_{i \leftarrow i}=0$. A naive block diagonal preconditioner $\mathcal{B}_{\mathrm{MPT}}$ can be constructed by taking the inverse of the diagonal blocks of $\mathcal{A}_{\mathrm{MPT}}$. However, as we demonstrated in [23], the resulting preconditioner is not robust with respect to variations in the conductivity and exchange parameters. In fact, the condition numbers increased linearly with the ratio between the exchange and conductivity coefficients.

3.2. Diagonalizing the MPT equations by congruence. In this section we discuss a reformulation of the MPT equations which, in turn, leads directly to a parameter-robust preconditioner. Let $P \in \mathbb{R}^{J \times J}$ be an invertible linear transformation defining a change of variables and let $\tilde{p}$ and $\tilde{q}$ be the new set of variables such that

$$
p=\left(P \otimes I_{W}\right) \tilde{p}, \quad q=\left(P \otimes I_{W}\right) \tilde{q}
$$

with $q=\left(q_{1}, q_{2}, \ldots, q_{J}\right)$ and similarly for $\tilde{q}, \tilde{p}$. Since $K$ and $E$ are symmetric, we apply Lemma 2.1 with $A=\Delta$ and $B=I_{W}$, to obtain a matrix, $P$, simultaneously diagonalizing $K$ and $E$ by congruence, that is, the equivalent operators $\left(P^{T} K P\right) \otimes \Delta$ and $\left(P^{T} E P\right) \otimes I_{W}$ are block diagonal. The resulting formulation (cf. (2.6)) of the MPT equations reads as follows: find the transformed pressures $\tilde{p}=\left(\tilde{p}_{1}, \ldots, \tilde{p}_{J}\right)$ such that, for a given $g \in Q$, we have the equality

$$
\tilde{\mathcal{A}}_{\mathrm{MPT}} \tilde{p}=\left(-\tilde{K} \otimes \Delta+\tilde{E} \otimes I_{W}\right) \tilde{p}=\left(P^{T} \otimes I_{W}\right) g,
$$

where $\tilde{K}=P^{T} K P$ and $\tilde{E}=P^{T} E P$ are diagonal with

$$
\tilde{K}=\operatorname{diag}\left(\tilde{K}_{1}, \ldots, \tilde{K}_{J}\right), \quad \tilde{E}=\operatorname{diag}\left(\tilde{\xi}_{1}, \ldots, \tilde{\xi}_{J}\right) .
$$

3.3. Preconditioning the transformed MPT system. The parameter dependent norm, for the transformed system, can be immediately identified [23] as

$$
\|\tilde{p}\|_{\tilde{\mathcal{B}}_{\mathrm{MPT}}}^{2}=\sum_{j=1}^{J}\left\langle\tilde{K}_{j} \nabla \tilde{p}_{j}, \nabla \tilde{p}_{j}\right\rangle+\left\langle\tilde{\xi}_{j} \tilde{p}_{j}, \tilde{p}_{j}\right\rangle .
$$

The associated preconditioner, arising from the above norm, for (3.5) is

$$
\tilde{\mathcal{B}}_{\mathrm{MPT}}=\left(\begin{array}{cccc}
\left(-\tilde{K}_{1} \Delta+\tilde{\xi}_{1} I\right)^{-1} & 0 & \cdots & 0 \\
0 & \left(-\tilde{K}_{2} \Delta+\tilde{\xi}_{2} I\right)^{-1} & \cdots & 0 \\
\vdots & \vdots & \ddots & \vdots \\
0 & 0 & \cdots & \left(-\tilde{K}_{J} \Delta+\tilde{\xi}_{J} I\right)^{-1}
\end{array}\right)
$$

Clearly, $\tilde{\mathcal{A}}_{\mathrm{MPT}}$ and $\tilde{\mathcal{B}}_{\mathrm{MPT}}^{-1}$ are trivially spectrally equivalent. We refer to [23] for numerical experiments comparing the standard and transformed formulation and preconditioners. 
3.4. Finding the transformation matrix. There are two cases that we will consider: the first case is when the matrix $C=K^{-1} E$ (cf. Theorem (A.2)) has $J$ distinct eigenvalues, while the second case will be for the case where at least one of the eigenvalues is repeated. In the case of distinct eigenvalues, the number of distinct eigenvalues of $C=K^{-1} E$ will depend on the material parameter values $K_{j}$ and $\xi_{j \rightarrow i}$ for $1 \leq i, j \leq J$. In the common case where $C$ has $J$ distinct eigenvalues, the transformation matrix is easily defined as follows. Let $\lambda_{1}, \ldots, \lambda_{J}$ be the real eigenvalues of $C$, and let $v_{1}, \ldots, v_{J}$ be the corresponding normalized eigenvectors. Then

$$
P=\left[v_{1}, \ldots, v_{J}\right]
$$

will diagonalize $K$ and $E$ by congruence. In [23], we presented numerical examples for the case of $J$ distinct eigenvalues (with $J=2$ ).

The congruence matrix for the case of repeated eigenvalues is also easily constructed. For these cases, the transform $P$ can be constructed by repeated application of blockwise eigenvector matrices; see [12] for the general procedure. In Example 3.1 below, we present an example on how to obtain the transformation matrix $P$ in the case where one of the eigenvalues has algebraic multiplicity 2 with $J=3$.

Example 3.1. In this example we show how to obtain the transformation matrix $P$ for a three-network case when one of the eigenvalues of $K^{-1} E$ has algebraic multiplicity 2. In this example, due to the presence of the repeated eigenvector, the construction of $P$ does not follow directly from the use of normalized eigenvectors and, thus, $P$ is not normalized a priori. We will, however, normalize $P$ following construction to maintain consistency with the previous case; in practice, either the normalized or nonnormalized version of $P$ may be used:

$$
K=\left(\begin{array}{ccc}
1.0 & 0 & 0 \\
0 & 0.0001 & 0 \\
0 & 0 & 0.01
\end{array}\right), \quad E=\left(\begin{array}{ccc}
1.01 & -0.01 & -1.0 \\
-0.01 & 0.0101 & -0.0001 \\
-1.0 & -0.0001 & 1.0001
\end{array}\right)
$$

By definition

$$
C=K^{-1} E=\left(\begin{array}{ccc}
1.01 & -0.01 & -1.0 \\
-100 & 101 & -1.0 \\
-100 & -0.01 & 100.01
\end{array}\right)
$$

The eigenvalues $\lambda_{1}, \lambda_{2}, \lambda_{3}$ and eigenvectors $\left[v_{1}, v_{2}, v_{3}\right]=P_{1}$ of $C$ are then

$$
\begin{aligned}
& \lambda_{1}=0, \quad \lambda_{2}=\lambda_{3}=101.01, \\
& P_{1}=\left(\begin{array}{ccc}
-0.5773 & -0.0071 & -0.0091 \\
-0.5773 & 0.7070 & -0.4031 \\
-0.5773 & 0.7070 & 0.9150
\end{array}\right) .
\end{aligned}
$$

In this specific case the eigenvalues $\lambda_{2}, \lambda_{3}$ have algebraic multiplicity 2 and geometrical multiplicity 1 . If we try to diagonalize $K$ and $E$ by congruence via $P_{1}$, we obtain

$$
\begin{aligned}
P_{1}^{T} K P_{1} & =\left(\begin{array}{ccc}
3.3670 \times 10^{-1} & 0 & 0 \\
0 & 5.1007 \times 10^{-3} & 6.5069 \times 10^{-3} \\
0 & 6.5069 \times 10^{-3} & 8.4729 \times 10^{-3}
\end{array}\right), \\
P_{1}^{T} E P_{1} & =101.01\left(\begin{array}{ccc}
0 & 0 & 0 \\
0 & 5.1007 \times 10^{-3} & 6.5069 \times 10^{-3} \\
0 & 6.5069 \times 10^{-3} & 8.4729 \times 10^{-3}
\end{array}\right) .
\end{aligned}
$$

Copyright $\odot$ by SIAM. Unauthorized reproduction of this article is prohibited. 
In this case, the resulting matrices are block diagonal. The lower right blocks are multiples of each other. We can diagonalize the lower right blocks by computing the eigendecomposition of either of these. The lower right block of $P_{1}^{T} K P_{1}$ is

$$
\left(\begin{array}{ll}
5.1007 \times 10^{-3} & 6.5069 \times 10^{-3} \\
6.5069 \times 10^{-3} & 8.4729 \times 10^{-3}
\end{array}\right)
$$

and its eigenpairs are

$$
\begin{aligned}
& \lambda_{1}=6.4967 \times 10^{-5}, \lambda_{2}=1.3508 \times 10^{-2}, \\
& P_{2}=\left(\begin{array}{cc}
-0.79083 & -0.6120 \\
0.6120 & -0.7908
\end{array}\right) .
\end{aligned}
$$

The final transformation matrix $P$ that diagonalizes $K$ and $E$ by congruence is then $(3.15)$

$$
P=P_{1}\left(\begin{array}{ccc}
1 & 0 & 0 \\
0 & & P_{2} \\
0 & &
\end{array}\right)=\left(\begin{array}{ccc}
-5.7735 \times 10^{-1} & 7.1935 \times 10^{-5} & 1.1575 \times 10^{-2} \\
-5.7735 \times 10^{-1} & -8.0594 \times 10^{-1} & -1.1391 \times 10^{-1} \\
-5.7735 \times 10^{-1} & 8.6590 \times 10^{-4} & -1.1564
\end{array}\right)
$$

Note that despite the columns of $P_{1}$ and $P_{2}$ being normalized with norm 1 , the resulting matrix $P$ 's columns are not normalized. After the normalization, the matrix $P$ looks as follows,

$$
P=\left(\begin{array}{ccc}
-5.7735 \times 10^{-1} & 8.9255 \times 10^{-5} & 9.9611 \times 10^{-2} \\
-5.7735 \times 10^{-1} & -9.9999 \times 10^{-1} & -9.8026 \times 10^{-2} \\
-5.7735 \times 10^{-1} & 1.0743 \times 10^{-4} & -9.9513 \times 10^{-1}
\end{array}\right)
$$

and the diagonalized matrices are as follows,

$$
\begin{aligned}
\tilde{K} & =P^{T} K P=\left(\begin{array}{ccc}
3.3670 \times 10^{-1} & 0 & 0 \\
0 & 1.0001 \times 10^{-4} & 0 \\
0 & 0 & 1.0003 \times 10^{-2}
\end{array}\right), \\
\tilde{E} & =P^{T} E P=\left(\begin{array}{ccc}
0 & 0 & 0 \\
0 & 1.0102 \times 10^{-2} & 0 \\
0 & 0 & 1.0104
\end{array}\right) .
\end{aligned}
$$

4. Preconditioning the MPET equations via diagonalization. In this section, we present a change of variables for the total pressure formulation of the timediscrete MPET equations; we propose and analyze a preconditioning strategy for the resulting variational formulation. The change of MPET variables is guided by the change of MPT variables presented in the previous section. The notation $Q$ signifies, as in section 3 , the $J$-fold product of the Hilbert space $W$.

4.1. Total pressure formulation of the MPET equations. Throughout this paper we assume the boundary conditions

$$
u=0 \text { on } \Gamma, \quad(2 \mu \varepsilon(u)+\lambda \operatorname{div} u \mathbb{I}) \nu=0 \quad \text { on } \partial \Omega \backslash \Gamma, \quad p_{j}=0 \text { on } \partial \Omega, j=1, \ldots, J,
$$

where $\nu$ is the unit outward normal vector field on $\partial \Omega$. The total pressure formulation of Biot's equations [16] and more generally the MPET equations [17] is a robust mixed variational formulation targeting the nearly incompressible limit $(\lambda \gg 1)$. The total pressure, which we will see satisfies $p_{0} \in L^{2}(\Omega)$, is defined by

$$
p_{0}=\lambda \operatorname{div} u-\alpha \cdot p
$$

Copyright (c) by SIAM. Unauthorized reproduction of this article is prohibited. 
where $^{1} \alpha=\left(\alpha_{1}, \ldots, \alpha_{J}\right) \in \mathbb{R}^{J}, p=\left(p_{1}, \ldots, p_{J}\right) \in Q$, and $\alpha \cdot p=\sum_{i=1}^{J} \alpha_{i} p_{i} \in W$. The total pressure formulation of (1.1) then reads as follows: for $t \in(0, T]$, find the displacement vector field $u$ and the pressure scalar fields $p_{0}$ and $p_{j}$ for $j=1, \ldots, J$ such that

$$
\begin{gathered}
-\operatorname{div}\left(2 \mu \varepsilon(u)+p_{0} \mathbb{I}\right)=f, \\
\operatorname{div} u-\lambda^{-1} p_{0}-\lambda^{-1} \alpha \cdot p=0, \\
\lambda^{-1} \dot{p}_{0}+s_{j} \dot{p}_{j}-\operatorname{div}\left(K_{j} \nabla p_{j}\right)+\alpha_{j} \lambda^{-1} \alpha \cdot \dot{p}+\sum_{i=1}^{J} \xi_{j \leftarrow i}\left(p_{j}-p_{i}\right)=g_{j}
\end{gathered}
$$

for $j=1, \ldots, J$.

We consider an implicit Euler discretization in time of the total pressure formulation of the time-dependent MPET equations (4.2) and examine the resulting stationary problem at each time step. The resulting time-discrete version of (4.2) with time step $\tau>0$ reads as follows: find the displacement $u$ and the pressures $p_{j}$ for $0 \leq j \leq J$ such that

$$
\begin{gathered}
-\operatorname{div}\left(2 \mu \varepsilon(u)+p_{0} \mathbb{I}\right)=f \\
\operatorname{div} u-\lambda^{-1} p_{0}-\lambda^{-1} \alpha \cdot p=0, \\
-s_{j} p_{j}-\alpha_{j} \lambda^{-1} p_{0}-\alpha_{j} \lambda^{-1} \alpha \cdot p+\tau \operatorname{div}\left(K_{j} \nabla p_{j}\right)-\tau \sum_{i=1}^{J} \xi_{j \leftarrow i}\left(p_{j}-p_{i}\right)=g_{j}
\end{gathered}
$$

for $1 \leq j \leq J$ where the new right-hand sides $g_{j}$ for $j=1, \ldots, J$ have been negated and also contain terms from the previous time step. Again, we impose homogeneous Dirichlet boundary conditions for all network pressures: $p_{j}=0$ on $\partial \Omega$ for $1 \leq j \leq J$.

Let $V=H_{\Gamma}^{1}\left(\Omega ; \mathbb{R}^{d}\right), Q_{0}=L^{2}(\Omega)$, and $Q_{j}=W=H_{0}^{1}(\Omega)$ for $1 \leq j \leq J$, and $\Omega \subset \mathbb{R}^{d}$. Let $Q=Q_{1} \times \cdots \times Q_{J}$. As in section 3 , we write $p=\left(p_{1}, \ldots, p_{J}\right)$, $q=\left(q_{1}, \ldots, q_{J}\right)$, and $g=\left(g_{1}, \ldots, g_{J}\right)$. Multiplying by test functions, and integrating second-order derivatives by parts, we obtain the following variational formulation of (4.3): find $u \in V$ and $p_{i} \in Q_{i}$ for $i=0, \ldots, J$ such that

$$
\begin{aligned}
a(u, v)+b\left(v, p_{0}\right) & =\langle f, v\rangle & & \forall v \in V, \\
b\left(u, q_{0}\right)-c_{1}\left(p_{0}, q_{0}\right)-c_{2}\left(q_{0}, p\right) & =0 & & \forall q_{0} \in Q_{0}, \\
-c_{2}\left(p_{0}, q\right)-c_{3}(p, q) & =\langle g, q\rangle & & \forall q \in Q .
\end{aligned}
$$

The bilinear forms $a: V \times V \rightarrow \mathbb{R}$ and $b: V \times Q_{0} \rightarrow \mathbb{R}$ are defined as

$$
a(u, v)=\langle 2 \mu \varepsilon(u), \varepsilon(v)\rangle, \quad b\left(v, q_{0}\right)=\left\langle\operatorname{div} v, q_{0}\right\rangle,
$$

while $c_{1}: Q_{0} \times Q_{0} \rightarrow \mathbb{R}, c_{2}: Q_{0} \times Q \rightarrow \mathbb{R}$, and $c_{3}: Q \times Q \rightarrow \mathbb{R}$ are defined as

$$
\begin{aligned}
c_{1}\left(p_{0}, q_{0}\right)= & \left\langle\lambda^{-1} p_{0}, q_{0}\right\rangle, \\
c_{2}\left(p_{0}, q\right)= & \left\langle\lambda^{-1} \alpha \cdot q, p_{0}\right\rangle, \\
c_{3}(p, q)= & \tau \sum_{j=1}^{J}\left\langle K_{j} \nabla p_{j}, \nabla q_{j}\right\rangle+\sum_{j=1}^{J}\left\langle s_{j} p_{j}, q_{j}\right\rangle \\
& +\tau \sum_{j=1}^{J} \sum_{i=1}^{J}\left\langle\xi_{j \leftarrow i}\left(p_{j}-p_{i}\right), q_{j}\right\rangle+\left\langle\lambda^{-1} \alpha \cdot p, \alpha \cdot q\right\rangle .
\end{aligned}
$$

For future reference we define $c:\left(Q_{0} \times Q\right) \times\left(Q_{0} \times Q\right) \rightarrow \mathbb{R}$ via

$$
c\left(\left(p_{0}, p\right),\left(q_{0}, q\right)\right)=c_{1}\left(p_{0}, q_{0}\right)+c_{2}\left(p_{0}, q\right)+c_{2}\left(q_{0}, p\right)+c_{3}(p, q) .
$$

\footnotetext{
${ }^{1}$ Note that we start counting at 1 in the definition of $p$ here and throughout, in contrast to, e.g., in [23].
}

Copyright (c) by SIAM. Unauthorized reproduction of this article is prohibited. 
4.2. MPET as a parameter-dependent saddle point system. Constructing parameter-robust block preconditioners for the system (4.4) is nontrivial. Here we demonstrate how the technique of diagonalization by congruence [23], [9, Remark 6] allows for easily extending the MPT preconditioning approach to that of the MPET system. Note that the system (4.3) or equivalently (4.4) can be viewed as a saddle point problem with a stabilization term (given by the bilinear form $c$ ). Thus, the equations fit well into Brezzi saddle point theory [4]. However, various material parameters in different ranges are involved in the system, so constructing parameter-robust preconditioners for this system is not a straightforward application of the Brezzi theory. Let us recall the parameter ranges we are concerned with in this paper. The existing literature covers the parameters

$$
0 \leq s_{j} \lesssim 1, \quad 0<K_{j} \ll 1, \quad 1 \lesssim \mu \lesssim \lambda<+\infty, \quad(1 \leq j \leq J) .
$$

In addition to these we are also interested in developing preconditioners which are robust for the ratios of the exchange coefficients $\xi_{i \rightarrow j}$ 's.

We first consider construction of preconditioners utilizing the saddle point problem structure. To reveal the saddle point problem structure of (4.4) let us look at the operator form of (4.4), which is

$$
\mathcal{A}_{\mathrm{MPET}}\left(\begin{array}{c}
u \\
p_{0} \\
p
\end{array}\right)=\left(\begin{array}{ccc}
-2 \operatorname{div}(\mu \varepsilon) & -\nabla & \mathbf{0} \\
\operatorname{div} & -C_{1} & -C_{2}^{*} \\
\mathbf{0} & -C_{2} & -C_{3}
\end{array}\right)\left(\begin{array}{c}
u \\
p_{0} \\
p
\end{array}\right)=\left(\begin{array}{l}
f \\
0 \\
g
\end{array}\right),
$$

where $C_{1}: Q_{0} \rightarrow Q_{0}^{*}, C_{2}: Q_{0} \rightarrow Q^{*}, C_{3}: Q \rightarrow Q^{*}$ are the operators associated with the bilinear forms $c_{1}, c_{2}, c_{3}$ in (4.6), (4.7), (4.8). Here $C_{2}^{*}$ is the adjoint operator of $C_{2}$. We can rewrite $\mathcal{A}_{\text {MPET }}$ of (4.10) in the standard saddle point form

$$
\mathcal{A}_{\mathrm{MPET}}=\left(\begin{array}{cc}
A & B_{0}^{*} \\
B_{0} & -C
\end{array}\right)
$$

by considering the product space grouping $V \times\left(Q_{0} \times Q\right)$ and identifying

$$
A=-2 \operatorname{div}(\mu \varepsilon), \quad B_{0}=(\operatorname{div}, \mathbf{0})^{T}, \quad C=\left(\begin{array}{ll}
C_{1} & C_{2}^{*} \\
C_{2} & C_{3}
\end{array}\right)
$$

One of the natural approaches to constructing block preconditioners for this system is to use the block diagonal operator

$$
\left(\begin{array}{cc}
A^{-1} & 0 \\
0 & \left(C+B_{0} A^{-1} B_{0}^{*}\right)^{-1}
\end{array}\right)
$$

or its approximation. However, the operator $\left(C+B_{0} A^{-1} B_{0}^{*}\right)^{-1}$ is not easy to implement efficiently in practice. Moreover, the analysis for spectral equivalence of this type of preconditioner is related to a nontrivial generalized eigenvalue problem. More precisely, the spectral equivalence is equivalent to uniform upper and lower bounds of the generalized eigenvalues, so it requires a deep analysis of the nontrivial generalized eigenvalue problem utilizing block matrix structures. In this paper we consider a general MPET model with general $J$ and general (constant) exchange coefficients, so the number of blocks in block matrices is not restricted. This makes an analysis of the generalized eigenvalue problem even more challenging, so we will not pursue this approach further in this paper. 
Another natural choice of block preconditioners for this system is a direct extension of the preconditioner in [16]. In other words, we use the block diagonal operator of the form

$$
\mathcal{B}_{\mathrm{MPET}}=\left(\begin{array}{ccc}
(-\mu \Delta)^{-1} & 0 & 0 \\
0 & I^{-1} & 0 \\
0 & 0 & D^{-1}
\end{array}\right),
$$

where $I: Q_{0} \rightarrow Q_{0}^{*}$ is the operator defined by the bilinear form $\left\langle p_{0}, q_{0}\right\rangle$ for $p_{0}, q_{0} \in Q_{0}$, $D: Q \rightarrow Q^{*}$ is the block diagonal operator such that its $j$ th diagonal block $(1 \leq j \leq J)$ is defined by selecting the $j$ th diagonal entry of the operator $C_{3}$ associated with the bilinear form (4.8), that is

$$
\tau\left\langle K_{j} \nabla p_{j}, \nabla q_{j}\right\rangle+\left\langle s_{j} p_{j}, q_{j}\right\rangle+\tau\left\langle\xi_{j} p_{j}, q_{j}\right\rangle+\left\langle\lambda^{-1} \alpha_{j} p_{j}, \alpha_{j} q_{j}\right\rangle, \quad p, q \in Q .
$$

However, this preconditioner is not robust with respect to the material parameters, particularly for the hydraulic conductivity and the exchange coefficients. We illustrate numerical experiment results in Example 4.1.

Example 4.1. Let $\Omega=[0,1]^{2} \subset \mathbb{R}^{2}$, and consider a structured triangulation $\mathcal{T}_{h}$ of $\Omega$ constructed by dividing $\Omega$ into $N \times N$ squares and then subdividing each square by a fixed diagonal. Let $J=2$. Consider a finite element discretization of (4.4) using the lowest order Taylor-Hood-type elements, i.e., continuous piecewise quadratics for each displacement component, and continuous piecewise linear for all pressures [17]. Let $\tau=1.0, \mu=1.0, s_{j}=1.0, \alpha_{j}=0.5$, and $K_{1}=1.0$, and consider ranges of values for $\lambda, \xi_{1 \leftarrow 2}$ and $K_{2}$. We consider the case for $s_{1}=s_{2}=1.0$ and $s_{1}=s_{2}=0.0$. Starting from an initial random guess, we consider a MinRes solver of the resulting linear system of equations with an algebraic multigrid (Hypre AMG) preconditioner of the form (4.12). The convergence criterion used was

$$
\left(\mathcal{B} r_{k}, r_{k}\right) /\left(\mathcal{B} r_{0}, r_{0}\right) \leq 10^{-6},
$$

where $r_{k}$ is the residual of the $k$ th iteration. The resulting number of Krylov iterations are shown in Table 2 for $\xi_{1 \rightarrow 2}=10^{6}$ and ranges of $K_{2}$ and $\lambda$. We observe that the number of iterations is moderate $(\approx 30)$ for $K_{2}$ of comparable magnitude $\left(10^{6}\right)$ to $\xi_{1 \leftarrow 2}$. The number of iterations increases with decreasing $K_{2}$ : up to $\approx 1000$ for $K_{2}=1$. For large $K_{2}$, the number of iterations seems independent of the mesh resolution $N$. In contrast, for smaller $K_{2}$ (relative to $\xi_{1 \rightarrow 2}$ ), the number of iterations also increases with the mesh resolution. We note that the iteration counts do not vary substantially with $\lambda$.

4.3. Diagonalizing the MPET equations by congruence. In this subsection, we present MPET equations which are transformed via change of variables for construction of block preconditioners. As in the MPT problem we will find an invertible linear map $P \in \mathbb{R}^{J \times J}$ that provides a fortuitous codiagonalization; we will then consider the change of variables

$$
p=\left(P \otimes I_{W}\right) \tilde{p},
$$

which will lead to a (partial) diagonalization, in the spirit of Lemma 2.1, for the transformed MPET system in the new unknowns $\left(u, p_{0}, \tilde{p}\right)$. For the discussions below let us give remarks on the block operators defined by $c_{1}, c_{2}, c_{3}$. Specifically, regarding $\alpha$ as a column vector,

$$
C_{2}=\left(\lambda^{-1} \alpha\right) \otimes I_{W}, \quad C_{3}=-\tau K \otimes \Delta+(S+\tau E+L) \otimes I_{W},
$$


TABLE 2

Number of MinRes iterations (cf. Example 4.1): (4.4) as discretized with Taylor-Hood-type elements and an algebraic multigrid preconditioner of the form (4.12). Of note is the fact that the number of iterations grows for $K_{2}$ decreasing relative to $\xi_{2 \rightarrow 1}=10^{6}$, and for increasing $N$.

\begin{tabular}{cc|cccc}
\hline$K_{2}$ & $\lambda$ & $N=16$ & 32 & 64 & 128 \\
\hline \multirow{4}{*}{$10^{0}$} & $10^{0}$ & 738 & 1271 & 1756 & 1938 \\
& $10^{2}$ & 1024 & 1505 & 1679 & 1631 \\
& $10^{4}$ & 1028 & 1506 & 1666 & 1628 \\
& $10^{6}$ & 1004 & 1499 & 1677 & 1633 \\
\hline \multirow{4}{*}{$10^{2}$} & $10^{0}$ & 396 & 424 & 406 & 353 \\
& $10^{2}$ & 337 & 368 & 351 & 333 \\
& $10^{4}$ & 364 & 352 & 348 & 332 \\
& $10^{6}$ & 345 & 357 & 361 & 328 \\
\hline \multirow{4}{*}{$10^{4}$} & $10^{0}$ & 65 & 65 & 62 & 60 \\
& $10^{2}$ & 64 & 60 & 56 & 55 \\
& $10^{4}$ & 62 & 60 & 57 & 55 \\
& $10^{6}$ & 63 & 61 & 58 & 55 \\
\hline \multirow{4}{*}{$10^{6}$} & $10^{0}$ & 30 & 30 & 30 & 28 \\
& $10^{2}$ & 34 & 31 & 29 & 29 \\
& $10^{4}$ & 32 & 31 & 31 & 29 \\
& $10^{6}$ & 33 & 31 & 31 & 29 \\
\hline
\end{tabular}

with $K$ and $E$ as in (3.1), $L$ is the matrix $L_{i j}=\lambda^{-1} \alpha_{i} \alpha_{j}, S$ is the diagonal matrix such that its $j$ th entry is $s_{j}$, and $I_{W}$ is the identity (functional) on $W$; we recall that $Q$ is the $J$-fold Cartesian product of $W$. We will first describe the transformed MPET equations for a general coordinate transformation, $P$. From the form of the transformed equations, we will extract the conditions for $P$ that yield a system that is suitable for the construction of parameter-robust block preconditioners.

Suppose we have a fixed but otherwise arbitrary, invertible coordinate transformation matrix $P \in \mathbb{R}^{J \times J}$. Applying this transformation of variables to the semidiscretized total pressure variational formulation of the MPET equations (4.4), we obtain the following variational formulation: find the displacement $u \in V$, the total pressure $p_{0} \in Q_{0}$, and the transformed pressures $\tilde{p}=\left(\tilde{p}_{1}, \ldots, \tilde{p}_{J}\right) \in Q$ such that

$$
\begin{aligned}
a(u, v)+b\left(v, p_{0}\right) & =\langle f, v\rangle & & \forall v \in V, \\
b\left(u, q_{0}\right)-c_{1}\left(p_{0}, q_{0}\right)-\tilde{c}_{2}\left(q_{0}, \tilde{p}\right) & =0 & & \forall q_{0} \in Q_{0}, \\
-\tilde{c}_{2}\left(p_{0}, \tilde{q}\right)-\tilde{c}_{3}(\tilde{p}, \tilde{q}) & =\left\langle g,\left(P \otimes I_{W}\right) \tilde{q}\right\rangle & & \forall \tilde{q} \in Q,
\end{aligned}
$$

where

$$
\tilde{c}_{2}\left(q_{0}, \tilde{q}\right) \equiv c_{2}\left(q_{0},\left(P \otimes I_{W}\right) \tilde{q}\right), \quad \tilde{c}_{3}(\tilde{p}, \tilde{q}) \equiv c_{3}\left(\left(P \otimes I_{W}\right) \tilde{p},\left(P \otimes I_{W}\right) \tilde{q}\right) .
$$

We define $\tilde{\mathcal{A}}_{\text {MPET }}: V \times Q_{0} \times Q \rightarrow\left(V \times Q_{0} \times Q\right)^{*}$ as the operator corresponding to the bilinear form (4.14). The operator form of the transformed system (4.14) then reads as

$$
\tilde{\mathcal{A}}_{\mathrm{MPET}}\left(\begin{array}{c}
u \\
p_{0} \\
\tilde{p}
\end{array}\right)=\left(\begin{array}{l}
f \\
0 \\
\tilde{g}
\end{array}\right), \quad \tilde{\mathcal{A}}_{\mathrm{MPET}}=\left(\begin{array}{ccc}
A & B^{T} & \mathbf{0} \\
B & -C_{1} & -\tilde{C}_{2} \\
\mathbf{0} & -\tilde{C}_{2} & -\tilde{C}_{3}
\end{array}\right),
$$

where $A=-2 \operatorname{div}(\mu \varepsilon), B=\operatorname{div}$ as before, and $\tilde{g}=\left(P^{T} \otimes I_{W}\right) g$. By inserting (4.13) 
and reordering, we note that

$$
\begin{aligned}
& \tilde{C}_{2}=P^{T} C_{2}=\left(\lambda^{-1} P^{T} \alpha\right) \otimes I_{W}, \\
& \tilde{C}_{3}=P^{T} C_{3} P=-\tau\left(P^{T} K P\right) \otimes \Delta+\left(P^{T} S P+P^{T}(\tau E+L) P\right) \otimes I_{W} .
\end{aligned}
$$

For simplicity we will write

$$
\tilde{\alpha}=\left(P^{T} \otimes I_{W}\right) \alpha
$$

We now look to apply Lemma 2.1 with the choice of operators $S=K \otimes \Delta$ and $T=(\tau E+L) \otimes I_{W}$. The matrices $K$ and $\tau E+L$ satisfy the required conditions and, thus, there exists (cf. Appendix A) an invertible transformation $P$ simultaneously diagonalizing $K$ and $\tau E+L$ by congruence. That is, we have matrices $\tilde{K}$ and $\tilde{\Gamma}$ given by the formulas

$$
\begin{aligned}
\tilde{K} & =P^{T} K P=\operatorname{diag}\left(\tilde{K}_{1}, \ldots, \tilde{K}_{J}\right), \\
\tilde{\Gamma} & =P^{T}(\tau E+L) P=\operatorname{diag}\left(\tilde{\gamma}_{1}, \ldots, \tilde{\gamma}_{J}\right) .
\end{aligned}
$$

We point out that the storage coefficients $\left\{s_{j}\right\}_{j=1}^{J}$ are not involved in this simultaneous diagonalization process. This is critically important in order to achieve a preconditioner that is parameter robust, even in the presence of vanishing storage coefficients. For future reference we briefly note that

$$
\tilde{\gamma}_{j} \geq \tilde{\alpha}_{j}^{2} / \lambda
$$

follows from the definition of $\tilde{\Gamma}$ in (4.19) since $E$ is positive semidefinite and therefore $\tilde{\gamma}_{j}$ is greater than or equal to the $j$ th diagonal entry of the matrix $\left(P^{T} L P\right)_{i j}=$ $\lambda^{-1} \tilde{\alpha}_{i} \tilde{\alpha}_{j}$.

We also remark that the following identity holds for $\tilde{c}_{3}$ :

$$
\begin{aligned}
\tilde{c}_{3}(\tilde{p}, \tilde{q})= & \left.\tau \sum_{j=1}^{J}\left\langle\tilde{K}_{j} \nabla \tilde{p}_{j}, \nabla \tilde{q}_{j}\right\rangle+\sum_{j=1}^{J}\left\langle s_{j}\left(\left(P \otimes I_{W}\right) \tilde{p}\right)\right)_{j},\left(\left(P \otimes I_{W}\right) \tilde{q}\right)_{j}\right\rangle, \\
& +\tau \sum_{j=1}^{J} \sum_{i=1}^{J}\left\langle\xi_{j \leftarrow i}\left(\left(\left(P \otimes I_{W}\right) \tilde{p}\right)_{j}-\left(\left(P \otimes I_{W}\right) \tilde{p}\right)_{i}\right),\left(\left(P \otimes I_{W}\right) \tilde{q}\right)_{j}\right\rangle \\
& +\left\langle\lambda^{-1} \tilde{\alpha} \cdot \tilde{p}, \tilde{\alpha} \cdot \tilde{q}\right\rangle,
\end{aligned}
$$

where $\left(\left(P \otimes I_{W}\right) \tilde{p}\right)_{j}$ is the $j$ th component of $\left(P \otimes I_{W}\right) \tilde{p}$.

4.4. Preconditioning of the transformed MPET system. In this subsection we show that a parameter-robust preconditioner can be constructed using an appropriate parameter-dependent norm.

We first define a parameter-dependent norm

$$
\left\|\left(u, p_{0}, \tilde{p}\right)\right\|_{\tilde{\mathcal{B}}}^{2}=\|\varepsilon(u)\|_{2 \mu}^{2}+\left\|p_{0}\right\|_{(2 \mu)^{-1}}^{2}+\sum_{j=1}^{J}\left\|\nabla \tilde{p}_{j}\right\|_{\tau \tilde{K}_{j}}^{2}+\sum_{j=1}^{J}\left\|\tilde{p}_{j}\right\|_{\tilde{\gamma}_{j}}^{2}
$$

and consider the associated block preconditioner of the form

$$
\tilde{\mathcal{B}}_{\text {MPET }}=\left(\begin{array}{ccccc}
(-2 \mu \Delta)^{-1} & 0 & 0 & \cdots & 0 \\
0 & 2 \mu I^{-1} & 0 & \cdots & 0 \\
0 & 0 & \left(-\tau \tilde{K}_{1} \Delta+\tilde{\gamma}_{1} I\right)^{-1} & \cdots & 0 \\
\vdots & \vdots & \vdots & \ddots & \vdots \\
0 & 0 & 0 & \cdots & \left(-\tau \tilde{K}_{J} \Delta+\tilde{\gamma}_{J} I\right)^{-1}
\end{array}\right) .
$$


Lemma 4.2 (continuity). Let $\tilde{\mathcal{A}}_{\mathrm{MPET}}$ be defined by (4.16), and consider the norm defined by (4.22). We assume that $2 \mu \leq M_{0} \lambda$ for some $M_{0}>0$ and $s_{j} \lesssim \tilde{\gamma}_{j}$ for $1 \leq j \leq J$. Then there exists a constant $C>0$, dependent on $M_{0}$, the constants in $s_{j} \lesssim \tilde{\gamma}_{j}$, the matrix $P$, but independent of any other problem parameters, such that

$$
\left\langle\tilde{\mathcal{A}}_{\mathrm{MPET}}\left(u, p_{0}, \tilde{p}\right),\left(v, q_{0}, \tilde{q}\right)\right\rangle \leq C\left\|\left(u, p_{0}, \tilde{p}\right)\right\|_{\tilde{\mathcal{B}}}\left\|\left(v, q_{0}, \tilde{q}\right)\right\|_{\tilde{\mathcal{B}}}
$$

for all $\left(u, p_{0}, \tilde{p}\right),\left(v, q_{0}, \tilde{q}\right) \in V \times Q_{0} \times Q$.

Proof. By redistributing the material parameter weights and the Cauchy-Schwarz inequality, we obtain the preliminary upper bound

$$
\left\langle\tilde{\mathcal{A}}_{\mathrm{MPET}}\left(u, p_{0}, \tilde{p}\right),\left(v, q_{0}, \tilde{q}\right)\right\rangle \leq Z_{1}+Z_{2}+Z_{3}=: Z,
$$

where

$$
\begin{aligned}
& Z_{1}=\|\varepsilon(u)\|_{2 \mu}\|\varepsilon(v)\|_{2 \mu}+\left\|p_{0}\right\|_{(2 \mu)^{-1}}\|\operatorname{div} v\|_{2 \mu}+\left\|q_{0}\right\|_{(2 \mu)^{-1}}\|\operatorname{div} u\|_{2 \mu}, \\
& Z_{2}=\left\|p_{0}\right\|_{\lambda^{-1}}\left\|q_{0}\right\|_{\lambda^{-1}}+\|\tilde{\alpha} \cdot \tilde{q}\|_{\lambda^{-1}}\left\|p_{0}\right\|_{\lambda^{-1}}+\|\tilde{\alpha} \cdot \tilde{p}\|_{\lambda^{-1}}\left\|q_{0}\right\|_{\lambda^{-1}}, \\
& Z_{3}=\sum_{j=1}^{J}\left(\left\|\nabla \tilde{p}_{j}\right\|_{\tau_{\tilde{K}_{j}}}\left\|\nabla \tilde{q}_{j}\right\|_{\tau_{\tilde{K}_{j}}}+\left\|\tilde{p}_{j}\right\|_{\tilde{\gamma}_{j}}\left\|\tilde{q}_{j}\right\|_{\tilde{\gamma}_{j}}+\left\langle s_{j}(P \tilde{p})_{j},(P \tilde{q})_{j}\right\rangle\right) .
\end{aligned}
$$

Since $\|\operatorname{div} u\| \leq\|\varepsilon(u)\|$ and by $2 \mu \leq M_{0} \lambda$ and the assumptions on $s_{j}$ and $\tilde{\gamma}_{j}$, it follows that

$$
\begin{aligned}
Z \lesssim & \left(\|\varepsilon(u)\|_{2 \mu}+\left\|p_{0}\right\|_{(2 \mu)^{-1}}+\|\tilde{\alpha} \cdot \tilde{p}\|_{\lambda^{-1}}+\sum_{j=1}^{J}\left(\left\|\nabla \tilde{p}_{j}\right\|_{\tau \tilde{K}_{j}}+\left\|\tilde{p}_{j}\right\|_{\tilde{\gamma}_{j}}\right)\right) \\
& \times\left(\|\varepsilon(v)\|_{2 \mu}+\left\|q_{0}\right\|_{(2 \mu)^{-1}}+\|\tilde{\alpha} \cdot \tilde{q}\|_{\lambda^{-1}}+\sum_{j=1}^{J}\left(\left\|\nabla \tilde{q}_{j}\right\|_{\tau \tilde{K}_{j}}+\left\|\tilde{q}_{j}\right\|_{\tilde{\gamma}_{j}}\right)\right) .
\end{aligned}
$$

By the triangle inequality and (4.20) we obtain

$$
\|\tilde{\alpha} \cdot \tilde{p}\|_{\lambda^{-1}} \leq \sum_{j=1}^{J}\left\|\tilde{p}_{j}\right\|_{\tilde{\alpha}_{j}^{2} / \lambda} \leq \sum_{j=1}^{J}\left\|\tilde{p}_{j}\right\|_{\tilde{\gamma}_{j}}
$$

and this completes the proof.

LEMma 4.3 (inf-sup condition). Let $\tilde{\mathcal{A}}_{\mathrm{MPET}}, \tilde{\mathcal{B}}_{\mathrm{MPET}}$, and all assumptions be as in Lemma 4.2. Then, there exists a constant $C>0$, dependent on $M_{0}$, the constants in $s_{j} \lesssim \tilde{\gamma}_{j}$, but independent of other material parameters, such that

$$
\inf _{\left(u, p_{0}, p\right)} \sup _{\left(v, q_{0}, q\right)} \frac{\left\langle\tilde{\mathcal{A}}_{\mathrm{MPET}}\left(u, p_{0}, p\right),\left(v, q_{0}, q\right)\right\rangle}{\left\|\left(u, p_{0}, p\right)\right\|_{\tilde{\mathcal{B}}}\left\|\left(v, q_{0}, q\right)\right\|_{\tilde{\mathcal{B}}}} \geq C,
$$

where the inf and sup are taken over the nonvanishing elements in $V \times Q_{0} \times Q$.

Proof. Consider any $\left(u, p_{0}, \tilde{p}\right) \in V \times Q_{0} \times Q$, and choose $\tilde{q}=-\tilde{p}$, and $q_{0}=-p_{0}$. Let $w \in V$ satisfy

$$
\left\langle\operatorname{div} w, p_{0}\right\rangle=\left\|p_{0}\right\|_{(2 \mu)^{-1}}^{2}, \quad\|\varepsilon(w)\|_{2 \mu} \leq C_{0}\left\|p_{0}\right\|_{(2 \mu)^{-1}}
$$

for a $C_{0}>0$ depending on the domain $\Omega$ via Korn's inequality, and next choose $v=u+2 \delta w$ for $\delta>0$ to be further specified. We note that, with this choice of $v, q_{0}$, and $q$,

$$
\left\|\left(v, q_{0}, \tilde{q}\right)\right\|_{\tilde{\mathcal{B}}} \lesssim\left\|\left(u, p_{0}, \tilde{p}\right)\right\|_{\tilde{\mathcal{B}}}
$$

Copyright $@$ by SIAM. Unauthorized reproduction of this article is prohibited. 
with the inequality constant depending only on the domain $\Omega$ and the choice of $\delta$ since

$$
\|\varepsilon(v)\|_{2 \mu} \leq\|\varepsilon(u)\|_{2 \mu}+2 \delta C_{0}\left\|p_{0}\right\|_{(2 \mu)^{-1}} \lesssim\left\|\left(u, p_{0}, \tilde{p}\right)\right\|_{\tilde{\mathcal{B}}} .
$$

Therefore, it suffices to show that

$$
\left\langle\tilde{\mathcal{A}}_{\mathrm{MPET}}\left(u, p_{0}, \tilde{p}\right),\left(v, q_{0}, \tilde{q}\right)\right\rangle \gtrsim\left\|\left(u, p_{0}, \tilde{p}\right)\right\|_{\tilde{\mathcal{B}}}^{2} .
$$

Using the definition of $\tilde{\mathcal{A}}_{\text {MPET }}$ together with (4.27), we find that

$$
\begin{aligned}
\left\langle\tilde{\mathcal{A}}_{\mathrm{MPET}}\left(u, p_{0}, \tilde{p}\right),\left(v, q_{0}, \tilde{q}\right)\right\rangle=\|\varepsilon(u)\|_{2 \mu}^{2}+ & 2 \delta\langle\varepsilon(u), \varepsilon(w)\rangle_{2 \mu}+2 \delta\left\|p_{0}\right\|_{(2 \mu)^{-1}}^{2} \\
& +c_{1}\left(p_{0}, p_{0}\right)+2 \tilde{c}_{2}\left(p_{0}, \tilde{p}\right)+\tilde{c}_{3}(\tilde{p}, \tilde{p}) .
\end{aligned}
$$

Note that $c_{1}\left(p_{0}, p_{0}\right)=\left\|p_{0}\right\|_{\lambda^{-1}}^{2}, \tilde{c}_{2}\left(p_{0}, \tilde{p}\right)=\left\langle\tilde{\alpha} \cdot \tilde{p}, p_{0}\right\rangle_{\lambda^{-1}}$, and

$$
\tilde{c}_{3}(\tilde{p}, \tilde{p})=\sum_{j=1}^{J}\left(\left\|\nabla \tilde{p}_{j}\right\|_{\tau \tilde{K}_{j}}^{2}+\left\|\tilde{p}_{j}\right\|_{\tilde{\gamma}_{j}}^{2}+\left\langle s_{j}(P \tilde{p})_{j},(P \tilde{p})_{j}\right\rangle\right)
$$

from the definitions of $\tilde{c}_{2}, \tilde{c}_{3}$, and the congruent diagonalization. Recall the inequality (4.25). Then we obtain

$$
\begin{aligned}
\delta & \left\|p_{0}\right\|_{(2 \mu)^{-1}}^{2}+c_{1}\left(p_{0}, p_{0}\right)+2 \tilde{c}_{2}\left(p_{0}, \tilde{p}\right)+\tilde{c}_{3}(\tilde{p}, \tilde{p}) \\
\geq & \delta\left\|p_{0}\right\|_{(2 \mu)^{-1}}^{2}+\left\|p_{0}\right\|_{\lambda^{-1}}^{2}+2\left\langle\tilde{\alpha} \cdot \tilde{p}, p_{0}\right\rangle_{\lambda^{-1}}+\sum_{j=1}^{J}\left(\left\|\tilde{p}_{j}\right\|_{\tilde{\gamma}_{j}}^{2}+\left\|\nabla \tilde{p}_{j}\right\|_{\tau \tilde{K}_{j}}^{2}\right) \\
\geq & \left(\delta / M_{0}+1\right)\left\|p_{0}\right\|_{\lambda^{-1}}^{2}+2\left\langle\tilde{\alpha} \cdot \tilde{p}, p_{0}\right\rangle_{\lambda^{-1}}+\sum_{j=1}^{J}\left(\left\|\tilde{p}_{j}\right\|_{\tilde{\gamma}_{j}}^{2}+\left\|\nabla \tilde{p}_{j}\right\|_{\tau_{\tilde{K}_{j}}}^{2}\right) \\
= & \left\|\sqrt{\delta / M_{0}+1} p_{0}+\tilde{\alpha} \cdot \tilde{p}\right\|_{\lambda^{-1}}^{2}-\left(\frac{\delta}{M_{0}}+1\right)^{-1}\|\tilde{\alpha} \cdot \tilde{p}\|_{\lambda^{-1}}^{2} \\
& +\sum_{j=1}^{J}\left(\left\|\tilde{p}_{j}\right\|_{\tilde{\gamma}_{j}}^{2}+\left\|\nabla \tilde{p}_{j}\right\|_{\tau \tilde{K}_{j}}^{2}\right) \\
\geq & \sum_{j=1}^{J}\left(\frac{\delta}{M_{0}+\delta}\left\|\tilde{p}_{j}\right\|_{\tilde{\gamma}_{j}}^{2}+\left\|\nabla \tilde{p}_{j}\right\|_{\tau \tilde{K}_{j}}^{2}\right),
\end{aligned}
$$

where the second inequality in the above follows from the assumption $2 \mu \leq M_{0} \lambda$ and the third inequality follows from (4.25). Thus,

$$
\begin{aligned}
& \left\langle\tilde{\mathcal{A}}_{\mathrm{MPET}}\left(u, p_{0}, \tilde{p}\right),\left(v, q_{0}, \tilde{q}\right)\right\rangle \\
& \quad \geq\|\varepsilon(u)\|_{2 \mu}^{2}+\delta\langle\varepsilon(u), \varepsilon(w)\rangle_{2 \mu}+\delta\left\|p_{0}\right\|_{(2 \mu)^{-1}}^{2}+\sum_{j=1}^{J}\left\|\nabla \tilde{p}_{j}\right\|_{\tau \tilde{K}_{j}}^{2} \\
& \quad+\frac{\delta}{M_{0}+\delta} \sum_{j=1}^{J}\left\|\tilde{p}_{j}\right\|_{\tilde{\gamma}_{j}}^{2} .
\end{aligned}
$$

On the other hand, the Cauchy-Schwarz inequality, the definition of $w$, and Young's inequality give that

$$
\delta\left|\langle\varepsilon(u), \varepsilon(w)\rangle_{2 \mu}\right| \leq \delta C_{0}\|\varepsilon(u)\|_{2 \mu}\left\|p_{0}\right\|_{(2 \mu)^{-1}} \leq \frac{1}{2}\|\varepsilon(u)\|_{2 \mu}^{2}+\frac{1}{2} \delta^{2} C_{0}^{2}\left\|p_{0}\right\|_{(2 \mu)^{-1}}^{2} .
$$

Inserting the negation of (4.31) as a lower bound in (4.30), we thus obtain that

$$
\begin{aligned}
& \left\langle\tilde{\mathcal{A}}_{\mathrm{MPET}}\left(u, p_{0}, \tilde{p}\right),\left(v, q_{0}, \tilde{q}\right)\right\rangle \\
& \geq \frac{1}{2}\|\varepsilon(u)\|_{2 \mu}^{2}+\delta\left(1-\frac{1}{2} \delta C_{0}^{2}\right)\left\|p_{0}\right\|_{(2 \mu)^{-1}}^{2}+\sum_{j=1}^{J}\left(\left\|\nabla \tilde{p}_{j}\right\|_{\tau \tilde{K}_{j}}^{2}+\frac{\delta}{M_{0}+\delta}\left\|\tilde{p}_{j}\right\|_{\tilde{\gamma}_{j}}^{2}\right) .
\end{aligned}
$$

By choosing $\delta$, in particular, e.g., by letting $\delta<2 / C_{0}^{2}$, the estimate (4.28) follows.

Copyright $@$ by SIAM. Unauthorized reproduction of this article is prohibited. 
Remark 4.4. In Lemmas 4.2 and 4.3 we assumed $s_{j} \lesssim \tilde{\gamma}_{j}$ for $1 \leq j \leq J$ and it covers the cases where the $s_{j}$ 's are degenerate. This assumption can be removed if $\lambda^{-1} \lesssim s_{j}, 1 \leq j \leq J$, hold with constants of scale 1 . For parameter-robust preconditioners we use $P$ which gives a different simultaneous diagonalization. More precisely, we consider $P$ satisfying (4.18), and $P^{T}(S+\tau E+L) P=\operatorname{diag}\left(\tilde{\gamma}_{1}, \ldots, \tilde{\gamma}_{J}\right)$ instead of (4.19). The norm (4.22) with these new $\tilde{K}_{j}$ 's and $\tilde{\gamma}_{j}$ 's, will be used to obtain parameter-robust preconditioners. Since the modification of proofs is straightforward and most steps are almost same, we omit the detailed proofs.

For concreteness, we here illustrate the form of the MPET equations and of the proposed preconditioner in a specific example.

Example 4.5. We consider the simple case of two networks with $K_{1}=K_{2}=1.0$, $s_{1}=s_{2}=1.0, \alpha_{1}=\alpha_{2}=0.5, \lambda=1.0, \xi_{1 \rightarrow 2}=0.0$, and $\tau=1.0$. The transformation matrix in this case is

$$
P=\frac{1}{\sqrt{2}}\left(\begin{array}{cc}
1 & -1 \\
1 & 1
\end{array}\right)
$$

We remark that $P$ is not normalized. The associated transformed MPET operator (cf. (4.16) and associated definitions), is then

$$
\tilde{\mathcal{A}}_{\text {MPET }}=\left(\begin{array}{cccc}
-2 \mu \operatorname{div} \varepsilon & -\nabla & 0 & 0 \\
\operatorname{div} & -\lambda^{-1} & -(\sqrt{2} \lambda)^{-1} & 0 \\
0 & -(\sqrt{2} \lambda)^{-1} & -\Delta+\frac{3}{2} & 0 \\
0 & 0 & 0 & -\Delta+1
\end{array}\right),
$$

and the proposed preconditioner will be in the following form:

$$
\tilde{\mathcal{B}}_{\text {MPET }}=\left(\begin{array}{cccc}
(-2 \mu \operatorname{div} \varepsilon)^{-1} & 0 & 0 & 0 \\
0 & (2 \mu)^{-1} & 0 & 0 \\
0 & 0 & (-\Delta+1)^{-1} & 0 \\
0 & 0 & 0 & (-\Delta+1)^{-1}
\end{array}\right) .
$$

The objective of this example was to illustrate the layout of the operators in a simple case. The results for more general numerical examples will be presented later.

\subsection{Numerical performance.}

Example 4.6. In this example we demonstrate the robustness of the block diagonal preconditioner (4.23) for a mixed finite element discretization of the transformed total pressure MPET equations (4.16). We consider the same test case, discretization, and solver setup as described in Example 4.1; the new preconditioner is the only modification. Parameter ranges are as follows: $K_{2} \in\left[10^{-6}, 10^{6}\right], \xi_{1 \leftarrow 2} \in\left[10^{-6}, 10^{6}\right]$, and $\lambda \in\left[1,10^{6}\right]$.

The resulting number of iterations is shown in Figure 1 for $K_{2} \in\left[10^{-6}, 1\right]$ and $\xi_{1 \leftarrow 2} \in\left[1,10^{6}\right]$ and $s_{1}=s_{2}=1.0$; omitted values demonstrated similar behaviors. Each of the subplots in Figure 1 represents a fixed choice of $K_{2}$ and $\xi_{1 \leftarrow 2}$. In each subplot four curves are shown; these curves show the number of MinRes iterations corresponding to different values of $\lambda$, indicated by their respective symbols, at discretization levels $N=16,32,64$, and 128. In Figure 2 we performed the same experiments with $s_{1}=s_{2}=0.0$.

For completeness we also performed numerical examples for $J=3,5,10$, and $K_{j}=1.0, s_{j}=1.0, \xi_{1 \leftarrow 2} \in\left[1,10^{6}\right]$, and $\lambda \in\left[1,10^{6}\right]$. Results are reported in Figure 3. 


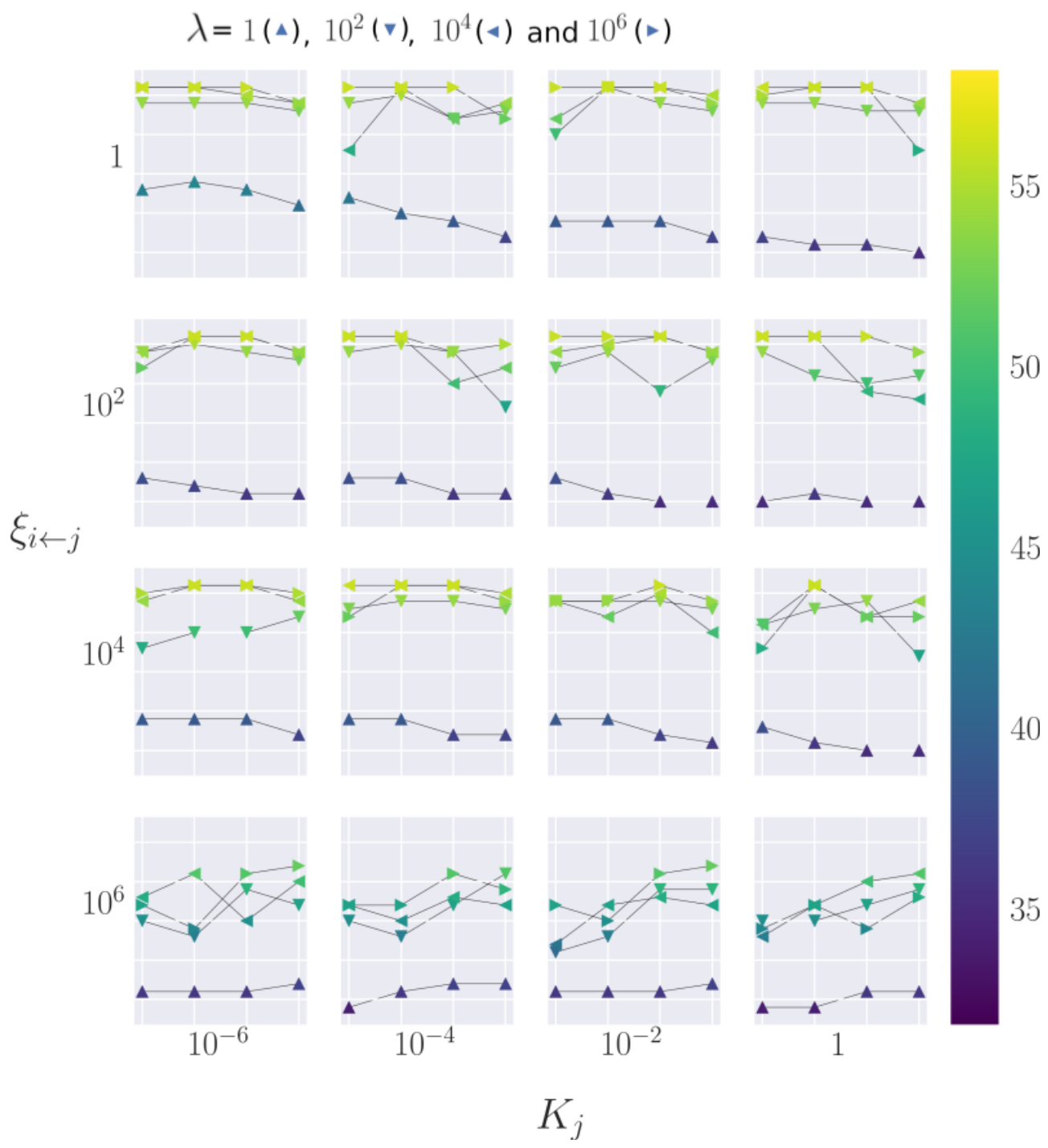

FIG. 1. Number of MinRes iterations: (4.4) discretized with Taylor-Hood-type elements and algebraic multigrid for $s_{1}=s_{2}=1.0 . K_{2}$ varies along the horizontal axis while the vertical axis shows variations in $\xi_{1 \leftarrow 2}$ for $K_{2}$ fixed. Each subplot contains four piecewise linear curves; each curve is decorated by a symbol indicating a corresponding value of $\lambda$ and corresponds to results for discretizations $N=16,32,64$, and 128 .

The stopping criterion was

$$
\left(\tilde{\mathcal{B}} r_{k}, r_{k}\right) /\left(\tilde{\mathcal{B}} r_{0}, r_{0}\right) \leq 10^{-6}
$$

where $r_{k}$ is the residual of the $k$ th iteration. We observe that the number of iterations is moderate in general. Moreover, the number of iterations does not grow for smaller $K_{2}$ 's relative to larger $\xi_{1 \leftarrow 2}$ or larger $N$ in contrast to what was observed for Example 4.1 .

Example 4.7. In this final example we present a modified version of a 3-dimensional (3D) footing problem [7, 24, 26]; we demonstrate the problem for two fluid 


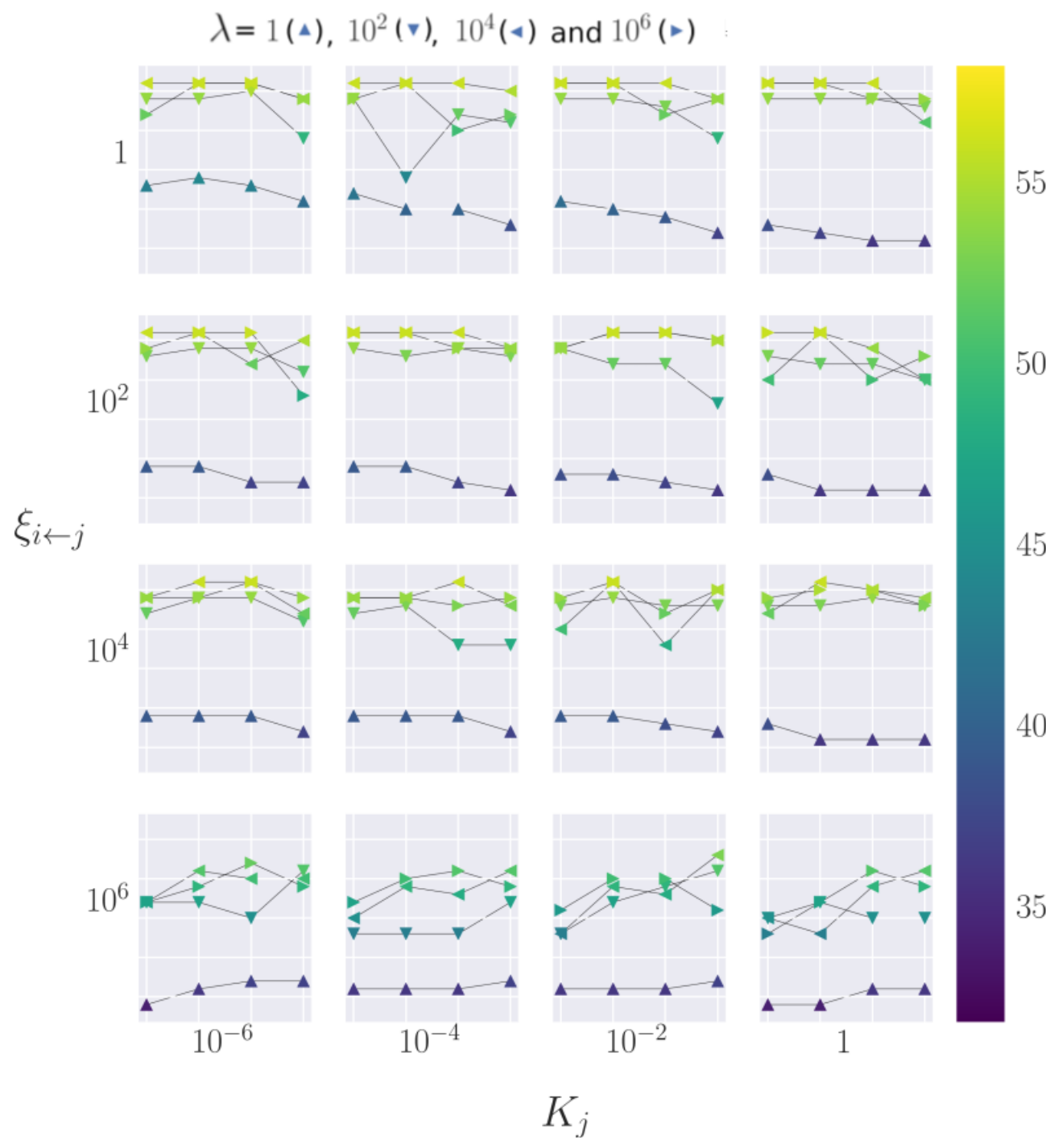

FIG. 2. Number of MinRes iterations: (4.4) discretized with Taylor-Hood-type elements and algebraic multigrid for $s_{1}=s_{2}=0 . K_{2}$ varies along the horizontal axis while the vertical axis shows variations in $\xi_{1 \leftarrow 2}$ for $K_{2}$ fixed. Each subplot contains four piecewise linear curves; each curve is decorated by a symbol indicating a corresponding value of $\lambda$ and corresponds to results for discretizations $N=16,32,64$, and 128 .

networks $(J=2)$ and use the standard unit cube, $\Omega=[0,1]^{3} \subset \mathbb{R}^{3}$, as computational domain. At the base of the domain, homogeneous Dirichlet conditions for the displacement and for both fluid pressures are imposed. At the topmost surface of the domain, i.e., $z=1$, a load of $0.1 \mathrm{~N} / \mathrm{m}^{2}$ is applied on the square $[0.25,0.75] \times[0.25,0.75]$, and a no flow condition is applied to the fluid pressures. For all remaining boundary sides of the domain, the zero stress condition is applied alongside a homogeneous Dirichlet condition for the fluid pressures. In the numerical experiments we vary the exchange coefficient $\xi_{1 \leftarrow 2}$, and the mesh size, the other physical parameters are reported in Table 3 .

In Table 4, we report the number of MinRes iterations for each time step (from 0.1 to 0.5 ), varying the mesh size and exchange parameters. The initial guess for the 

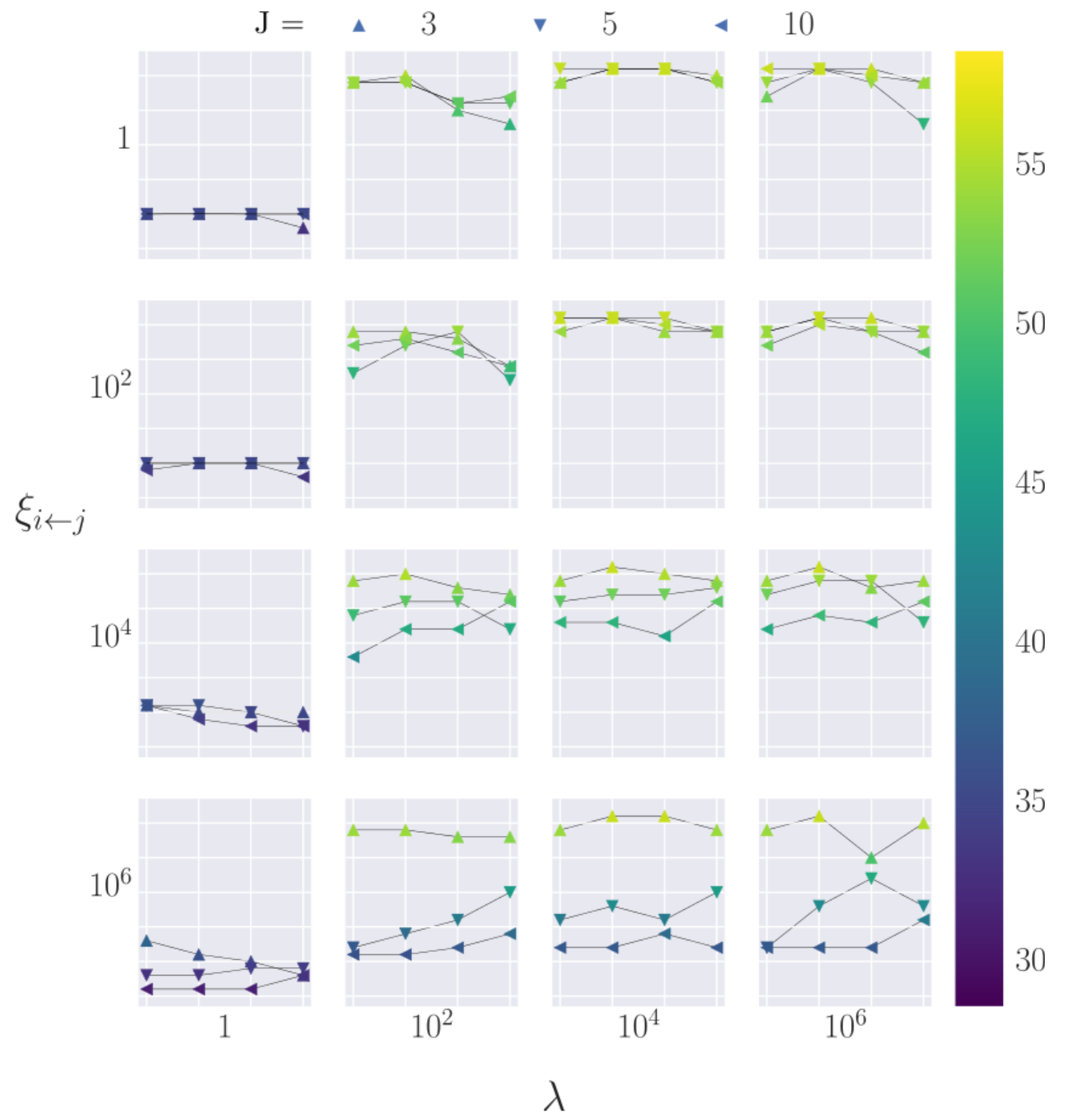

10

FIG. 3. Number of MinRes iterations: (4.4) for $J=3,5,10$ discretized with Taylor-Hood-type elements and algebraic multigrid for $K_{j}=1.0, s_{j}=1.0 . \lambda$ varies along the horizontal axis while the vertical axis shows variations in $\xi_{1 \leftarrow 2}$. Each subplot contains one piecewise linear curve and corresponds to results for discretizations $N=16,32,64$, and 128 .

TABLE 3

Parameters used in the numerical simulations.

\begin{tabular}{llll} 
Property & Symbol & Value & Units \\
\hline Young's modulus & $E$ & $3 \times 10^{4}$ & $\mathrm{~Pa}$ \\
Poisson ratio & $\nu$ & 0.45 & {$[-]$} \\
Hydraulic conductivities & $K_{1}, K_{2}$ & $10^{-6}$ & $\mathrm{~m}^{2}(\mathrm{~Pa} \mathrm{~s})^{-1}$ \\
Storage coefficients & $s_{1}, s_{2}$ & 0.0 & $\mathrm{~Pa}^{-1}$ \\
Biot coefficient & $\alpha_{1}, \alpha_{2}$ & 0.5 & {$[-]$} \\
\hline
\end{tabular}

solution is set to zero. Similarly to what observed in Example 4.6, the number of iterations is moderate also for this $3 \mathrm{D}$ case. In Figure 4 the solution for $t=0.5$ is shown.

Copyright $@$ ㅇ by SIAM. Unauthorized reproduction of this article is prohibited. 
TABLE 4

MinRes iterations for the footing problem (cf. Example 4.7).

\begin{tabular}{l|l|l|l|l|l|l}
\hline \multirow{2}{*}{$h$} & \multirow{2}{*}{$\xi_{1 \leftarrow 2}$} & \multicolumn{5}{|c}{ Number of iterations } \\
\cline { 3 - 7 } & & $t=0.1$ & $t=0.2$ & $t=0.3$ & $t=0.4$ & $t=0.5$ \\
\hline \multirow{2}{*}{$1 / 8$} & $1.0 \times 10^{-6}$ & 87 & 97 & 97 & 97 & 97 \\
& 1.0 & 89 & 102 & 102 & 102 & 102 \\
\hline \multirow{2}{*}{$1 / 16$} & $1.0 \times 10^{-6}$ & 90 & 102 & 102 & 102 & 102 \\
& 1.0 & 93 & 108 & 109 & 107 & 109 \\
\hline \multirow{2}{*}{$1 / 32$} & $1.0 \times 10^{-6}$ & 95 & 107 & 107 & 107 & 107 \\
& 1.0 & 98 & 112 & 112 & 114 & 111 \\
\hline
\end{tabular}

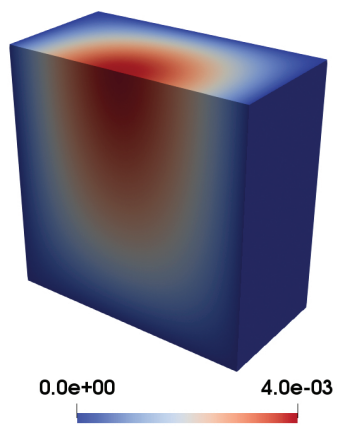

(a) fluid pressure $p_{1}$

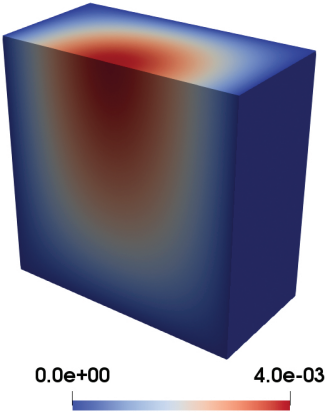

(b) fluid pressure $p_{2}$

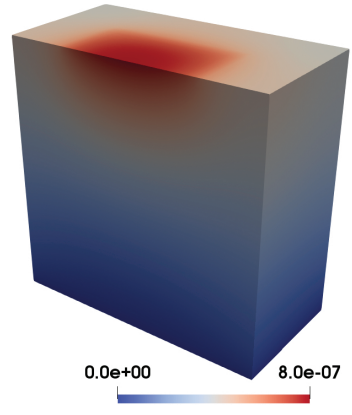

(c) displacement magnitude $u$

FIG. 4. A 3D Footing Problem, solution for $1 / h=32, \xi_{1 \leftarrow 2}=1.0$, at $t=0.5$.

5. Conclusions. In this paper, we have presented a new strategy for decoupling the total pressure variational formulation of the multiple-network poroelasticity equations. The decoupling strategy is based on a transformation via a change of variables, allowing for simultaneous diagonalization by congruence of the equation operators. In particular, the transformed equations are readily amenable for block diagonal preconditioning. Moreover, we have proposed a block diagonal preconditioner for the transformed system and shown theoretically that the preconditioner and the equation operator are norm equivalent, independently of the material parameters, under reasonable parameter assumptions. The theoretical results are supported by numerical examples. Combined, these results allow the efficient iterative solution of the multiple-network poroelasticity equations, even in the case of nearly incompressible materials.

We note that our strategy is based on spatially constant material parameters. The applicability of this approach for spatially varying parameters has not yet been considered.

Appendix A. Proof of Lemma 2.1. We first recall a basic [12] definition and result for posterity.

Definition A.1. A matrix $C \in \mathbb{C}^{n \times n}$ is diagonalizable if there exists an invertible transformation, $P$, such that $P^{-1} C P$ is diagonal. The matrix $C$ is called diagonalizable by congruence if there exists $P$, not necessarily invertible, such that $P^{T} C P$ is diagonal.

Theorem A.2 (see $\left[12,4.5 .17 \mathrm{a}-\mathrm{b}\right.$ p. 287]). Suppose $A$ and $B \in \mathbb{C}^{n \times n}$ are symmetric and that $A$ is invertible. Then $A$ and $B$ are diagonalizable by congruence if and only if $C=A^{-1} B$ is diagonalizable. 
Proof of Lemma 2.1. Assume $K$ and $E$ satisfy the hypotheses of Lemma 2.1 and, without loss of generality, suppose that $K$ is positive definite. Then $K$ is invertible and we first show that this implies $C=K^{-1} E \in \mathbb{R}^{n \times n}$ is diagonalizable. We note that $C$ satisfies

$$
K^{1 / 2} C K^{-1 / 2}=K^{-1 / 2} E K^{-1 / 2},
$$

where $K^{1 / 2}$ denotes the (unique, symmetric) principle square root of $K$. The righthand side, above, is symmetric due to the symmetry of $K$ and $E$. Thus $C$ is similar to a real, symmetric matrix and is therefore diagonalizable. From [12, 4.5.17a-b] there exists an invertible matrix $P \in \mathbb{R}^{n \times n}$ such that

$$
P^{T} K P=\tilde{D}_{K} \quad \text { and } \quad P^{T} E P=\tilde{D}_{E}
$$

where $\tilde{D}_{K}, \tilde{D}_{E} \in \mathbb{R}^{n \times n}$ are diagonal matrices.

Recalling $Q=W \times \cdots \times W$, define the change of variables $\tilde{p}=\left(P^{-1} \otimes I_{W}\right) p$ for $p \in Q$ and substitute into (2.5) to get

$$
\left\langle S\left(P \otimes I_{W}\right) \tilde{p}, q\right\rangle+\left\langle T\left(P \otimes I_{W}\right) \tilde{p}, q\right\rangle=\langle f, q\rangle \quad \forall q \in Q .
$$

Writing $q=\left(P \otimes I_{W}\right)\left(P^{-1} \otimes I_{W}\right) q$ and noting that the adjoint operator of $P \otimes I_{W}$ is $P^{T} \otimes I_{W^{*}}$, we have

$$
\left\langle S\left(P \otimes I_{W}\right) \tilde{p}, q\right\rangle=\left\langle\left(P^{T} \otimes I_{W^{*}}\right) S\left(P \otimes I_{W}\right) \tilde{p},\left(P^{-1} \otimes I_{W}\right) q\right\rangle .
$$

Since $S=K \otimes A$ we can obtain $\left(P^{T} \otimes I_{W^{*}}\right) S\left(P \otimes I_{W}\right)=\tilde{D}_{K} \otimes A$ (by the Hadamard product). By a similar argument

$$
\langle T P \tilde{p}, q\rangle=\left\langle\left(\tilde{D}_{E} \otimes B\right) \tilde{p},\left(P^{-1} \otimes I_{W}\right) q\right\rangle .
$$

Then $D_{S}:=\tilde{D}_{K} \otimes A$ and $D_{T}:=\tilde{D}_{E} \otimes B$ are block diagonal operators from $Q$ to $Q^{*}$. Finally $\langle f, q\rangle=\left\langle\left(P^{T} \otimes I_{W^{*}}\right) f,\left(P^{-1} \otimes I_{W}\right) q\right\rangle$ and the variational problem (2.6) follows because $q \in Q$ is arbitrary.

Remark A.3. The construction of the matrix $P$, yielding both $P^{T} K P=D_{1}$ and $P^{T} E P=D_{2}$, is straightforward for the case when $C=K^{-1} E$ has $n$ distinct eigenvalues. In this case $C$ has $n$ linearly independent eigenvectors; if $\left\{v_{1}, \ldots, v_{n}\right\}$ denote these eigenvectors then $P=\left[v_{1}, \ldots, v_{n}\right]$ is the matrix whose $j$ th column is $v_{j}$. When the eigenvalues of $C$ are not distinct, $P$ can be realized as the product of blockwise eigenvector matrices. The general procedure for this case is discussed in [12]; an example has been discussed in section 3. As a point of praxis it should be noted that that diagonalization by congruence can face a practical challenge. In particular, if the eigenvalues of $C=K^{-1} E$ are very large and the eigenvectors of $C$ have one or more small entries then computing the transformation matrix $P$, using off-the-shelf methods in, e.g., MATLAB or Mathematica, can be inexact. In such cases, the matrices $P^{T} K P$ and $P^{T} E P$ are strongly diagonally dominant but may have off diagonal entries that are approximately zero. In this case, alternative methods to compute the eigenvector matrix $P$ may be useful. For instance, one may consider using a package, such as the Python mpmath package, that supports variable precision to compute the eigenvectors and transformation matrices. 


\section{REFERENCES}

[1] M. Bai, D. Elsworth, And J.-C. Roegiens, Multiporosity/multipermeability approach to the simulation of naturally fractured reservoirs, Water Res. Res., 29 (1993), pp. 1621-1633.

[2] M. A. Biot, General theory of three-dimensional consolidation, J. Appl. Phys., 12 (1941), pp. $155-164$.

[3] J. BRašnová, V. Lukeš, And E. Rohan, Identification of multi-compartment Darcy flow model material parameters, in 20th International Conference on Applied Mechanics 2018, Myslovice, University of West Bohemia, Pilsen, Czech Republic, 2019, pp. 9-13.

[4] F. BrezzI, On the existence, uniqueness and approximation of saddle-point problems arising from Lagrangian multipliers, RAIRO Numer. Anal., 8 (1974), pp. 129-151.

[5] D. Chou, J. C. Vardakis, L. Guo, B. J. Tully, and Y. Ventikos, A fully dynamic multicompartmental poroelastic system: Application to aqueductal stenosis, J. Biomech., 49 (2016), pp. 2306-2312.

[6] A. EISENTRÄGER AND I. Sobey, Multi-fluid poroelastic modelling of CSF flow through the brain, in Poromechanics V: Proceedings of the Fifth Biot Conference on Poromechanics, American Society of Civil Engineers, Reston, VA, 2013, pp. 2148-2157.

[7] F. Gaspar, J. Gracia, F. Lisbona, and C. Oosterlee, Distributive smoothers in multigrid for problems with dominating grad-div operators, Numer. Linear Algebra Appl., 15 (2008), pp. 661-683, https://doi.org/10.1002/nla.587.

[8] Q. Hong And J. Kraus, Parameter-robust stability of classical three-field formulation of Biot's consolidation model, Electron. Trans. Numer. Anal., 48 (2018), pp. 202-226, https://doi. org/10.1553/etna_vol48s202.

[9] Q. Hong, J. Kraus, M. Lymbery, and F. Philo, Conservative discretizations and parameterrobust preconditioners for Biot and multiple-network flux-based poroelasticity models, Numer. Linear Algebra Appl., 26 (2019), e2242.

[10] Q. Hong, J. Kraus, M. Lymbery, and F. Philo, Parameter-robust Uzawa-type iterative methods for double saddle point problems arising in Biot's consolidation and multiplenetwork poroelasticity models, Math. Models. Methods. Appl. Sci., 30 (2020), pp. 25232555.

[11] Q. Hong, J. Kraus, M. Lymbery, and M. F. Wheeler, Parameter-robust convergence analysis of fixed-stress split iterative method for multiple-permeability poroelasticity systems, Multiscale Model. Simul., 18 (2020), pp. 916-941, https://doi.org/10.1137/19M1253988.

[12] R. A. Horn and C. R. Johnson, Matrix Analysis, 2nd ed., Cambridge University Press, 1990.

[13] T. Józsa, W. El-Bouri, R. Padmos, S. Payne, and A. Hoekstra, A cerebral circulation model for in silico clinical trials of ischaemic stroke, in The Proceedings of the CompBioMed Conference 2019.

[14] J. Lee, A. Cookson, R. Chabiniok, S. Rivolo, E. Hyde, M. Sinclair, C. Michler, T. Sochi, AND N. Smith, Multiscale modelling of cardiac perfusion, in Modeling the Heart and the Circulatory System, Springer, Cham, Switzerland, 2015, pp. 51-96.

[15] J. J. LeE, Unconditionally Stable Second Order Convergent Partitioned Methods for MultipleNetwork Poroelasticity, preprint, https://arxiv.org/abs/1901.06078 (2019).

[16] J. J. Lee, K.-A. Mardal, And R. Winther, Parameter-robust discretization and preconditioning of Biot's consolidation model, SIAM J. Sci. Comput., 39 (2017), pp. A1-A24.

[17] J. J. Lee, E. Piersanti, K.-A. Mardal, and M. E. Rognes, A mixed finite element method for nearly incompressible multiple-network poroelasticity, SIAM J. Sci. Comput., 41 (2019), pp. A722-A747.

[18] K.-A. Mardal And R. Winther, Preconditioning discretizations of systems of partial differential equations, Numer. Linear Algebra Appl., 18 (2011), pp. 1-40.

[19] C. Michler, A. Cookson, R. Chabiniok, E. Hyde, J. Lee, M. Sinclair, T. Sochi, A. Goyal, G. Vigueras, D. Nordsletten, and N. P. Smith, A computationally efficient framework for the simulation of cardiac perfusion using a multi-compartment Darcy porous-media flow model, Int. J. Numer. Methods Biomed. Eng., 29 (2013), pp. 217-232.

[20] A. Mikelić And M. F. Wheeler, Convergence of iterative coupling for coupled flow and geomechanics, Comput. Geosci., 17 (2013), pp. 455-461, https://doi.org/10.1007/ s10596-012-9318-y.

[21] M. J. M. Mokhtarudin and S. J. Payne, The study of the function of AQP4 in cerebral ischaemia-reperfusion injury using poroelastic theory, Int. J. Numer. Methods Biomed. Eng., 33 (2017), e02784.

[22] R. OYARzÚA AND R. RUIZ-BAIER, Locking-free finite element methods for poroelasticity, SIAM J. Numer. Anal., 54 (2016), pp. 2951-2973.

Copyright $@$ by SIAM. Unauthorized reproduction of this article is prohibited. 
[23] E. Piersanti, M. E. Rognes, And K.-A. Mardal, Parameter robust preconditioning for multicompartmental Darcy equations, in EnuMath 2019, Springer, Cham, Switzerland, 2021, pp. $703-711$.

[24] C. Rodrigo, X. Hu, P. Ohm, J. Adler, F. Gaspar, and L. Zikatanov, New stabilized discretizations for poroelasticity and the Stokes' equations, Comput. Methods Appl. Mech. Engrg., 341 (2018), pp. 467-484, https://doi.org/10.1016/j.cma.2018.07.003.

[25] R. Shipley, P. Sweeney, S. Chapman, and T. Roose, A four-compartment multiscale model of fluid and drug distribution in vascular tumours, Int. J. Numer. Methods Biomed. Eng. 36, (2020), e3315.

[26] E. Storvik, J. Both, K. Kuman, J. Nordbotten, and F. Radu, On the optimization of the fixed-stress splitting for Biot's equations, Internat. J. Numer. Methods. Engrg., 120 (2019), pp. 179-194, https://doi.org/10.1002/nme.6130.

[27] K. H. Støverud, M. Alnes, H. P. Langtangen, V. Haughton, and K.-A. Mardal, Poroelastic modeling of syringomyelia - a systematic study of the effects of pia mater, central canal, median fissure, white and gray matter on pressure wave propagation and fluid movement within the cervical spinal cord, Comput. Methods Biomech. Biomed. Eng., 19 (2016), pp. 686-698.

[28] B. Tully And Y. Ventikos, Modelling normal pressure hydrocephalus as a "two-hit" disease using multiple-network poroelastic theory, in ASME 2010 Summer Bioengineering Conference, American Society of Mechanical Engineers Digital Collection, Reston, VA. 2013, pp. $877-878$.

[29] B. J. Tully And Y. Ventikos, Cerebral water transport using multiple-network poroelastic theory: Application to normal pressure hydrocephalus, J. Fluid Mech., 667 (2011), pp. 188215.

[30] J. C. Vardakis, D. Chou, B. J. Tully, C. C. Hung, T. H. Lee, P.-H. Tsui, and Y. Ventikos, Investigating cerebral oedema using poroelasticity, Med. Eng. Phys., 38 (2016), pp. 48-57.

Copyright (c) by SIAM. Unauthorized reproduction of this article is prohibited. 